\title{
Applications of Atomic Ensembles in Distributed Quantum Computing
}

\author{
Marcin Zwierz and Pieter Kok \\ Department of Physics \& Astronomy, \\ The University of Sheffield \\ Hounsfield Road, Sheffield \\ S3 7RH United Kingdom \\ php07mz@sheffield.ac.uk
}

June 27, 2010

\begin{abstract}
Thesis chapter. The fragility of quantum information is a fundamental constraint faced by anyone trying to build a quantum computer. A truly useful and powerful quantum computer has to be a robust and scalable machine. In the case of many qubits which may interact with environment and their neighbors, protection against decoherence becomes quite a challenging task. The scalability and decoherence issues are the main difficulties addressed by the distributed model of quantum computation. A distributed quantum computer consists of a large quantum network of distant nodes - stationary qubits which communicate via flying qubits. Quantum information can be transferred, stored, processed and retrieved in decoherence-free fashion by nodes of a quantum network realized by an atomic medium an atomic quantum memory. Atomic quantum memories have been developed and demonstrated experimentally in recent years. With a help of linear optics and laser pulses one is able to manipulate quantum information stored inside an atomic quantum memory by means of electromagnetically induced transparency and associated propagation phenomena. Any quantum computation or communication necessarily involves entanglement. Therefore, one must be able to entangle distant nodes of a distributed network. In this article, we focus on the probabilistic entanglement generation procedures such as well-known DLCZ protocol. We also demonstrate theoretically a scheme based on atomic ensembles and the dipole blockade mechanism for generation of inherently distributed quantum states so-called cluster states. In
\end{abstract}


the protocol, atomic ensembles serve as single qubit systems. Hence, we review single-qubit operations on qubit defined as collective states of atomic ensemble. Our entangling protocol requires nearly identical single-photon sources, one ultra-cold ensemble per physical qubit, and regular photodetectors. The general entangling procedure is presented, as well as a procedure that generates in a single step $Q$-qubit GHZ states with success probability $p_{\text {success }} \sim \eta^{Q / 2}$, where $\eta$ is the combined detection and source efficiency. This is significantly more efficient than any known robust probabilistic entangling operation. The GHZ states form the basic building block for universal cluster states, a resource for the one-way quantum computer.

\section{Introduction}

An atomic ensemble or atomic vapor is a gas that consists of several hundred of the same species of atoms, typically alkali atoms such as Cesium or Rubidium, trapped at room temperature or trapped and cooled to $\mu \mathrm{K}$ temperature. An atomic ensemble may serve as a good interface between light and matter but has to meet the following requirements. The atoms have to possess a long lived ground state that is easily populated by optical pumping techniques. Moreover, the macroscopic ensemble should have a large optical depth $d=\rho \sigma L$, where $\rho$ is the atom number density, $\sigma$ is the absorption cross section of an atom and $L$ denotes the length of atomic medium. In other words, the atomic ensemble should easily, i.e., effectively, interact with light pulses. This is in fact one of the main advantages of atomic ensembles for interface purposes. A large number of atoms increases the coupling strength of an interaction between light and matter, and therefore allow us to coherently manipulate the quantum state of the ensemble with light and vice versa. Moreover, a large number of atoms helps to suppress the negative impact of decoherence on information stored in an atomic ensemble. $[1,2,3,4,5]$

The simplest way to prepare an atomic ensemble is to trap a cloud of alkali atoms in a glass cell. This is the so-called hot atomic vapor or room temperature atomic vapor. The room temperature atomic ensembles are used extensively because of their simplicity and large optical depth, which is the key figure of merit for quantum memory efficiency. These kinds of interfaces will inherently suffer from thermal motion and therefore the Doppler broadening. Moreover, atoms moving in and out of the interaction region may limit the performance of a quantum memory. One of the widely used methods to overcome this problem is utilization of a buffer gas. [6, 7] A few 
torr of a nobel gas, typically neon or helium, limits the thermal diffusion of an atoms inside a vapor.[7, 8] Another advantage of a buffer gas is the suppression of decoherence from the collisions between alkali atoms and with the walls of a cell. By means of a buffer gas the atoms can retain coherence for more than $10^{8}$ collisions. [6] Although a buffer gas seems to be indispensable, too high buffer gas pressure may also introduce some incoherent processes to the operation of a quantum memory.[6] One of the most recent techniques for suppression of the collisional and motional decoherence involves buffer gas cooled below $7 \mathrm{~K}$. In an experiment by Hong et al.[9], Rubidium atoms are cooled by a buffer gas and the diffusion time is slowed down. Moreover, the optical depth of a medium in this experiment is very large $(d>$ 70). The mentioned setup combines simplicity and large optical depth of a room temperature atomic vapor with slow atomic motion that is characteristic for another technique of trapping alkali atoms, namely so-called magneto-optical trapping (MOT).[9]

A MOT technique combines laser cooling and trapping with magnetic fields. Atoms trapped with MOT are cooled down to the $\mu \mathrm{K}$ temperature, therefore the collisional and motional decoherence becomes negligible in comparison with a typical operational time scale of a quantum interface. The shortcoming of a cold atomic ensemble is rather low optical depth (between 1 and 10). The very principle on which the MOT is operating, i.e., the magnetic field, also introduces another difficulty. The magnetic field causes decoherence of the ground states usually realized as a magnetic Zeeman sublevels of a ground state. This problem can be overcome by switching off the MOT trap and then performing operations on a quantum memory.[2] However, lack of the magnetic field trapping allows atoms to slowly diffuse and therefore limits the lifetime of a quantum interface. Nevertheless, by means of the MOT trap atomic vapors can be prepared in the form of a "frozen" gas with lifetime much longer than in the case of a room temperature vapors.

The last widely used method for confining large numbers of atoms to a small sample is called Bose-Einstein condensation. A Bose-Einstein condensate (BEC) has extremely large optical depth, however the preparation of a BEC is a highly demanding experiment.

There are a number of effects that influence the overall efficiency of quantum memories based on atomic ensembles. In spite of many efforts the efficiency of quantum memories reaches at the best 70\%.[2] The main source of low fidelity is a low optical depth $d$. Only optically thick medium, i.e., highly dense and/or large medium, can effectively interact with the light fields. The inefficiency of storage of light pulses based on techniques such as electromagnetically induced transparency or Raman interaction scales as 
$1 / d$.[2] The broadening, both homogenous and inhomogeneous, is another source of decoherence for quantum interfaces. The homogenous broadening is due to the spontaneous emission and depends on the optical depth as $1 / d$. For atomic ensembles at room temperature the inhomogeneous broadening is due to the Doppler broadening, which acts on all atoms in completely incoherent fashion and scales as $1 / d_{\text {broad }}^{2}$, where $d_{\text {broad }}$ is the optical depth in a presence of the homogenous broadening. For a sufficiently dense and/or large medium, inhomogeneous broadening is less dominant than homogenous broadening. Atomic motion and therefore atomic collisions are another factor that limits fidelity of a quantum memory.

The paper is organized as follows. In Sec. 2, we review in some detail a well known technique for coherent manipulation of atomic ensembles, namely electromagnetically induced transparency. Electromagnetically induced transparency and Raman interaction are important phenomena associated with interactions between atomic vapors and light fields. In the same section, we review the concept of an atomic medium as single-photon quantum memory. In Sec. 3, we introduce a notion of the Rydberg state and the dipole blockade mechanism which also may be used to induce coherent behavior of a macroscopic atomic medium. In Sec. 4 we review several schemes for probabilistic entanglement generation between atomic vapors such as DLCZ protocol and double-heralding protocol. In Sec. 5, we review the concept of an atomic ensemble as single qubit system and analyze in detail a scheme for single-qubit operations in atomic ensembles. In Sec. 6, we give a description of a new entangling operation and consider its usefulness for generation of the GHZ and cluster states. In Sec. 7, we consider all major errors and decoherence mechanisms that enter the entangling procedure and propose several experimental implementations.

\section{Electromagnetically induced transparency}

Electromagnetically induced transparency (EIT) is one of the most widely used techniques associated with quantum interfaces, i.e., interactions between atomic vapors and optical pulses. The well known techniques from quantum optics and atomic physics can be used for a coherent manipulation of the quantum states of light and matter. One of the most important and interesting phenomena in quantum optics is electromagnetically induced transparency, a term coined by Harris et al. in 1990.[10, 11] Its importance stems from the range of new potential applications it promises for nonlinear optics and quantum information applications. EIT is a phenom- 
ena resulting from the modification of the optical properties of an atomic medium, i.e., atomic ensemble, driven by an optical laser pulse. $[3,4,12]$ The laser field induces coherent behavior of an atomic medium and leads to the vanishing absorption and rapidly varying refraction of a resonant signal field [Fig. 2].[13, 14] The optical properties of atomic medium, i.e., linear response, are determined by the first-order linear susceptibility $\chi^{(1)}$. The imaginary part of the susceptibility $\operatorname{Im}\left[\chi^{(1)}\right]$ represents the absorption of the optical field by a medium and the real part $\operatorname{Re}\left[\chi^{(1)}\right]$ represents the refractive index $n$.

The optical proprieties of any atomic medium are mostly determined by its level structure.[3] The behavior of a two-level atomic medium in the presence of a resonant optical pulse seems rather straightforward. The laser pulse induces Rabi oscillations, i.e., atomic population is transferred between two levels in a coherent fashion. Addition of a single level to the level structure of atoms dramatically changes this picture. This slight change leads to a number of new and non-intuitive phenomena such as appearance of dark state polaritons and EIT itself. The phenomenon of EIT is based on quantum interference in the amplitudes of excitation pathways which results in destructive interference of the imaginary part of the linear susceptibility. In other words, at resonance linear response of a medium is canceled and the atomic medium is completely transparent to the signal field. The idea of interference between different excitation channels was first introduced by Fano.[3] Apart from the transparency window, number of new possibilities emerge such as opportunity to "stop" a light pulse inside a medium.

To understand the essence of EIT, let us consider an ensemble of atoms with a $\Lambda$-type three-level structure driven by two optical fields. Each atom in an ensemble has a pair of lower, long lived energy states $|g\rangle$ and $|s\rangle$. These states can be realized by the electronic ground state of alkali atoms and the transition between them is always dipole-forbidden. A state $|g\rangle$ is coupled to an excited state $|e\rangle$ through the signal optical field. A second strong control field is applied to the transition between state $|s\rangle$ and $|e\rangle$ [Fig. 1]. In this setting, the only way to absorption is by means of the $|e\rangle$ level. The EIT understood as a lack of the absorption emerges by means of destructive quantum interference between different absorption pathways - the direct one $|g\rangle-|e\rangle$ and the indirect pathways such as $|g\rangle-|e\rangle-|s\rangle-|e\rangle .[3]$ If the control field is much stronger than a signal field and both are detuned by the same amount, amplitudes of these different pathways have the same magnitude but the opposite sign and cancel each other.[3] In this picture atoms are said to be in a so-called dark superposition of the states $|g\rangle$ and $|s\rangle$, which leads to vanishing light absorption. Let us examine the Hamiltonian of the atomic 


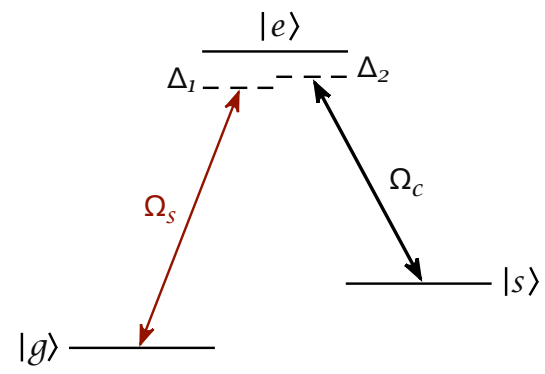

Figure 1: The three-level $\Lambda$-type scheme for EIT. $|g\rangle$ and $|s\rangle$ are a lower, long lived energy states and $|e\rangle$ is an excited state. $\Omega_{s}$ is the Rabi frequency of the signal field detunned from the atomic transition by $\Delta_{1}=\omega_{e g}-\omega_{s} . \Omega_{c}$ is the Rabi frequency of the control field detunned from the atomic transition by $\Delta_{2}=\omega_{e s}-\omega_{c}$.

$\Lambda$-type three-level system driven by a pair of near-resonant optical fields. In terms of the Hamiltonian $\hat{H}$, the system can be described as a sum of the free evolution atom Hamiltonian $\hat{H}_{0}$ and the interaction Hamiltonian $\hat{H}_{\text {int }}$. [3] Within the dipole approximation and in the rotating wave approximation, the interaction Hamiltonian $\hat{H}_{\text {int }}$ is given by

$$
\hat{H}_{i n t}=-\frac{\hbar}{2}\left[\begin{array}{ccc}
0 & 0 & \Omega_{s} \\
0 & -2\left(\Delta_{1}-\Delta_{2}\right) & \Omega_{c} \\
\Omega_{s} & \Omega_{c} & -2 \Delta_{1}
\end{array}\right],
$$

where $\Omega_{s}$ is the Rabi frequency of the signal field with frequency $\omega_{s}$ detuned from the corresponding atomic transition by $\Delta_{1}=\omega_{e g}-\omega_{s}$ and $\Omega_{c}$ is the Rabi frequency of the control field with frequency $\omega_{c}$ detunned from the corresponding atomic transition by $\Delta_{2}=\omega_{e s}-\omega_{c}$. [3] The dynamics of the system as whole is captured by the Hamiltonian $\hat{H}$. For two-photon resonance $\left(\Delta_{1}=\Delta_{2}=\Delta\right)$, the Hamiltonian $\hat{H}$ has a set of three eigenstates. In terms of the bare atom states $|g\rangle,|s\rangle$ and $|e\rangle$, one of the eigenstates has the form $|\psi(\theta)\rangle=\cos \theta|g\rangle-\sin \theta|s\rangle$, where $\theta$ is the so-called mixing angle given by $\tan \theta=\Omega_{s} / \Omega_{c}$. [3, 15] Under a two-photon resonance the $|\psi\rangle$ is a stationary state. The $|\psi\rangle$ is called a dark state because it has no contribution from $|e\rangle$, hence there is no possibility of absorption. Consequently, an opaque atomic medium, i.e., optically thick, is completely transparent to the signal field in a presence of a strong control field. In general, appearance of the transparency is independent of the detuning $\Delta$ of the signal optical field but, as one would expect, the larger $\Delta$ the narrower, in terms of a signal 
field frequency, is the transparency window.[3] Naturally, the ideal transparency occurs at the exact resonance. However, the increased control field strength can circumvent the limitations that are imposed by the resonance condition and even away from the resonance transparency can be observed. The reader should note an interesting feature of the dark state: $|\psi\rangle$ depends on the mixing angle $\theta$. This opens a route to extraordinary possibilities and applications.

\subsection{Stimulated Raman adiabatic passage}

The state of a system described above can be easily manipulated with an appropriate change of Rabi frequencies of the signal and control optical fields. [3] An adiabatic evolution known as stimulated Raman adiabatic passage (STIRAP) can be applied to the system to prepare it in a dark state $|\psi\rangle$. The STIRAP technique is governed by the interaction Hamiltonian $\hat{H}_{\text {int }}$ given above with $\Delta_{1}=\Delta_{2}=\Delta$. The adiabatic passage starts when $\Omega_{s} \ll \Omega_{c}$ and the system is in the ground state $|\psi\rangle=|g\rangle$. Then $\Omega_{s}$ is adiabatically increased and $\Omega_{c}$ is adiabatically decreases up to the point when $\sin \theta=1(\cos \theta=0)$ and the dark state $|\psi\rangle=-|s\rangle$. Consequently, by choosing appropriate Rabi frequency for both optical fields, it is possible to transfer a whole atomic population to a maximally coherent dark state $|\psi\rangle=1 / \sqrt{2}(|g\rangle-|s\rangle)$.[3] More importantly the STIRAP technique is immune to spontaneous emission losses since an excited state $|e\rangle$ is never populated, and therefore the number of photons in the optical field is conserved. The STIRAP technique allow us to prepare the system in one of the bare states $(|g\rangle$ or $|s\rangle)$ and in any intermediate superposition. Hence, the STIRAP procedure is a widely used technique for quantum state preparation in atomic ensembles.

\subsection{The propagation phenomena}

The EIT technique modifies not only the optical properties of an atomic medium, the propagation of optical pulses inside a medium is affected as well. These special propagation effects are the source for a variety of applications. First of all, the group velocity of a signal field, i.e., the velocity of the envelope of a wave packet, is changed.[16] Under EIT conditions, the group velocity, is reduced since the refractive index $n$ is varying rapidly in the neighborhood of the two-photon resonance as shown in Fig. 2 (the derivative of the refractive index with respect to the frequency is positive and large) 
and

$$
\nu_{g r}=\frac{c}{n+\frac{d n}{d \omega} \omega}=\frac{c}{1+n_{g r}},
$$

with $n_{g r} \sim \rho \sigma c / \Omega_{c}^{2}$ is the group index and $\sigma=3 \lambda^{2} / 2 \pi$ is the absorption cross section of an atom and $\rho$ is the atom number density.[3] Moreover, at
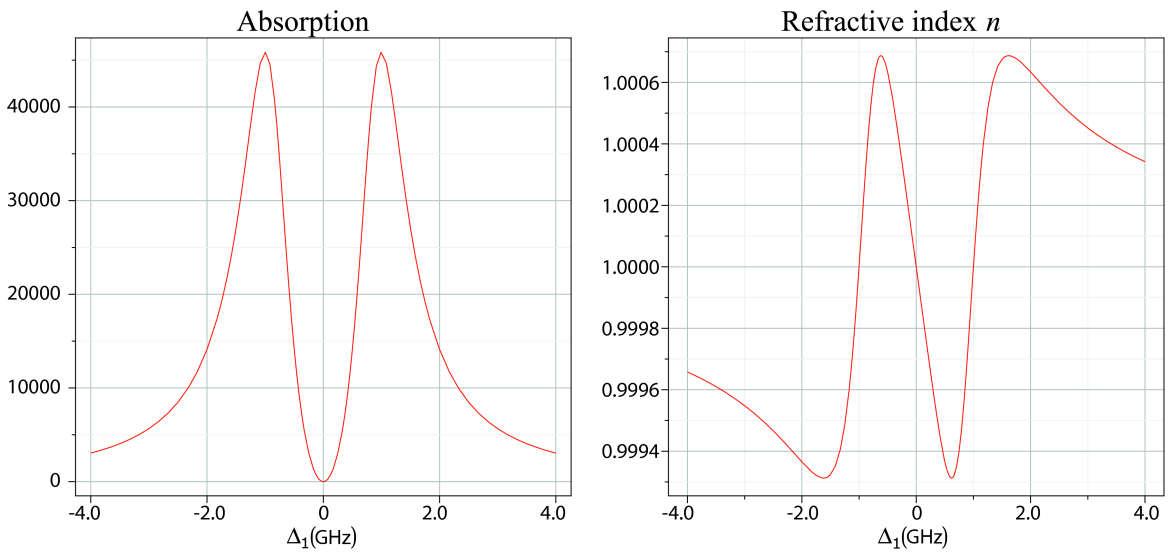

Figure 2: Absorption coefficient $\left(\operatorname{Im}\left[\chi^{(1)}\right]\right)$ and refractive index $n\left(\operatorname{Re}\left[\chi^{(1)}\right]\right)$ of the optical signal field interacting with an atomic medium in the presence of strong control field. $\Delta_{1}$ is the detuning between the signal field and the atomic transition. The strong control field is on resonance with the appropriate atomic transition, i.e., $\Delta_{2}=0$. The above figures were prepared with a help of chapter on atomic ensembles in quantum information processing in Ref. [17].

resonance the refractive index is equal to unity therefore the phase velocity, i.e., the velocity of a phase front, is equal to the speed of light in vacuum c. For high atomic densities and low Rabi frequency of the control field, the group velocity can be lowered to very small values. Different scientific groups performed experiments in which slow group velocities were obtained. In some of the experiments an ultra-cold and dense vapor $\mathrm{Na}$ atoms were used[18], in others light pulse was stopped in a hot Rb vapor[19] or even in solids. [20] The most remarkable result was obtained in an experiment by Hau et al. where the optical pulse was slowed to $17 \mathrm{~m} / \mathrm{s}$ in a Bose-Einstein condensate of $\mathrm{Na}$ atoms. [21] Naturally, all these experiments suffer from low transfer and storage efficiency due to decoherence effects that are intrinsic to the atomic system. All challenges concerning the atomic vapors that are 
encountered by experimentalists were described in the previous section. One may ask if it is possible to fully stop the optical pulse in the medium, i.e., "freeze it". Unfortunately, the decreasing group velocity leads to the decreasing transparency window which at some point vanishes and absorption of the signal pulse occurs again. To overcome this limitation one may use a non-stationary, time-dependent control field which dynamically narrows the frequency spectrum of a signal pulse proportional to the group velocity. [3] In other words, the group velocity has to be reduced adiabatically and this allows for the frequency spectrum of the signal pulse to reside within the transparency window. As one would expect, the trapping of the signal pulse, i.e., gradual reduction of the control field intensity, should commence when the entire pulse is within the medium which requires $T_{\text {signal }}<L / \nu_{g r}$ to avoid leakage of the front edge of the signal pulse.[4] This again require an optically dense atomic medium. The fact that one can slow down and confine an entire optical pulse in atomic ensemble for some time may seem unheard-of. Although, this effect is much more comprehensible when viewed from the point of view of an atomic medium. During a slowdown of an optical pulse many additional and interesting effects happen. The reduced velocity introduces a time delay of the light pulse in an atomic medium $\tau_{d}=n_{g r} L / c \sim \rho \sigma L$ and a spatial compression of the signal pulse in the propagation direction. The longer propagation time may be very advantageous in the case of non-linear medium enhancing the non-linear effects. The time delay is proportional to the optical depth of a medium $d=\rho \sigma L$, therefore substantial time delay requires optically thick medium. The spatial compression is associated with different propagation velocities inside and outside the medium. The front edge of a pulse propagates in the medium with different velocity than its back edge that propagates outside of the medium with the velocity $c$. This gives rise to the spatial compression by a ratio of the group velocity to the speed of light outside the atomic ensemble.[3, 4] The spatial compression means that the part of photons from the signal pulse is temporarily stored in the medium in the form of excitations. It is important to point out that no energy carried by photons is stored in the medium only the quantum state of light and the excess energy is transferred to the control field. [4] This process resembles the stimulated Raman passage. When the optical pulse enters the medium the total number of photons is reduced and the state of atomic system is adiabatically changed to a superposition between the bare states $|g\rangle$ or $|s\rangle$. When the pulse starts to leave the medium this process is reversed. The atomic excitations are turned back to the signal photons with a help of the control field and the state of the system comes back to the bare state $|g\rangle$. Naturally, this adiabatic process depends on the 
strength of the control field.

All these effects associated with slow light propagation can also be analyzed from the point of view of the atomic medium. Under these conditions, a system consisting of an atomic medium driven by optical fields can be described by introducing a new quantum field $\hat{\Psi}(z, t)$ that is a coherent mixture of electromagnetic and atomic excitations $(|g\rangle-|s\rangle$ excitation).[3, 15] The field $\hat{\Psi}(z, t)$ has a form

$$
\begin{gathered}
\hat{\Psi}(z, t)=\cos \theta \hat{E}(z, t)-\sin \theta \sqrt{N} \hat{S}(z, t), \\
\cos \theta=\frac{\Omega_{c}}{\sqrt{\Omega_{c}^{2}+\Omega_{s}^{2}}}, \quad \sin \theta=\frac{\Omega_{s}}{\sqrt{\Omega_{c}^{2}+\Omega_{s}^{2}}},
\end{gathered}
$$

where $\hat{E}(z, t)=\sum_{k} \hat{a}_{k}(t) e^{i k z}$ is the electric field operator of the signal field consisted of the free-space modes with bosonic operators $\hat{a}_{k}$ and wave vectors $k, \hat{S}(z, t)=1 / \sqrt{N} \sum_{i=1}^{N}\left|g_{i}\right\rangle\left\langle s_{i}\right| e^{-i \omega_{g s} t}$ corresponds to the atomic wave where $N$ is the number of atoms in the medium and $\omega_{g s}$ is the frequency between long-lived levels $|g\rangle$ and $|s\rangle$. The excitation of the field is called a polariton. The field $\hat{\Psi}(z, t)$ obeys the wave equation:

$$
\left[\frac{\partial}{\partial t}+c \cos ^{2} \theta \frac{\partial}{\partial z}\right] \hat{\Psi}(z, t)=0
$$

and propagates with group velocity $\nu_{g r}=c \cos ^{2} \theta$. By gradually changing the intensity of the control field one can modify the properties of the polariton from electromagnetic $\hat{E}(z, t)$, with propagation velocity close to the speed of light $c$, to purely atomic $\hat{S}(z, t)$ with propagation velocity close to zero. It is important to stress that for low group velocities not all but almost all photons from the optical pulse are transferred to the atomic medium. The character of the polariton depends on the intensity of control field and density of atomic medium. In other words, when control field strength is adiabatically lowered, the signal field is transferred to the atomic medium and propagates as an atomic wave, therefore in some sense the signal pulse is "stopped". After some time this "write" process can be reversed. When an intensity of the control field is increased, the signal pulse is retrieved from the atomic medium. Consequently, under EIT conditions the atomic medium acts as a quantum memory capable of slowing down, storing and releasing optical laser pulses or even single photon wave packets with high fidelity. The EIT enables to reverse the storing procedure and retrieve written information. Most importantly, since the transfer and retrieval of the light field is an adiabatic and coherent process, all proprieties of the light pulse are conserved at all times. The applications of electromagnetically 
induced transparency, i.e., the stopping of light pulses by means of the stimulated Raman adiabatic passage, for quantum information processing seem natural. This technique gives capability for coherent transfer of quantum states between optical light fields and matter system such as atomic vapors. Therefore, EIT can be used for preparation of specific nonclassical and entangled states of atomic medium.[22]

\subsection{Atomic medium as single-photon quantum memory}

The EIT and all associated phenomena can also be observed for singlephoton wave packets. Hence, one can imagine a single-photon coherently mapped onto atomic medium. [23, 24] The subsequent state of a medium is described by symmetric and collective atomic state given by

$$
|s\rangle=\frac{1}{\sqrt{N}} \sum_{j=1}^{N}\left|g_{1}, g_{2}, \ldots, s_{j}, \ldots, g_{N}\right\rangle,
$$

with high fidelity.[23] The $|s\rangle$ is a coherent W state. The optically dense EIT medium may serve as a good quantum memory that can be employed as a node in quantum network or quantum repeater.[25, 26] All atoms in a medium have the same probability of absorbing a single photon. This implies a very attractive feature of the collective state: it is impossible to learn which atom really absorbed a photon. [4] Therefore, within small error the loss of one atom has no effect on the fidelity of resulting state. This remarkable property of collective states make them very robust with respect to decoherence and losses.[4] In general, EIT based quantum memory is capable of storing not only single photon states but any superposition of photonic states, e.g. an entangled state. Although, the EIT based applications for quantum information science are very promising, one has to remember about many potential limitation associated with atomic based quantum memories. In many experiential trials, it has been proved that for high transfer-storage-retrieval efficiency, one has to use an atomic ensemble with a very large optical depth, i.e., high density of atoms or large sample size.[7] Consequently, higher density will introduce stronger collisional and dephasing effects, which are one of the most severe decoherence effects.[27, 28] Collisions during write and read processes may substantially limit the fidelity of quantum memory.[6] One way of dealing with the de-

coherence processes such as collisions and diffusion is exploitation of a cold atomic vapors in strong optical traps. Other ways are specific, entangled states of light as input fields and optimal input pulse shapes.[7, 8, 27] 
In spite of many difficulties, recent advances in quantum memories are remarkable. In recent experiments, truly quantum, optical memories that preserve quantum features of light such as entanglement have been demonstrated.[29, 30,31 In these experiments, a quantum memory was fed by a non-classical field of light originating from an atomic ensemble. An atomic ensemble serving as a source is prepared by a weak laser pulse so that only one of the atoms is in the excited state. This is so-called weak excitation regime. The excited atom relaxes to one of the lower energy levels emitting a single photon, the so-called Stokes photon that carries less energy than the absorbed photon. Next, the strong retrieve laser pulse brings the atomic medium back to its ground state and atomic excitation is converted into anti-Stokes photon.[2, 30, 31] The described technique proved to be extremely useful not only as a way of generating single-photon pulses but in many different applications such as the quantum repeater protocol, i.e., the DLCZ protocol. Subsequently, the nonclassical character of the source was verified with a help of the correlation function. Conditional on detection of one Stokes photon, after the retrieve pulse one observes either no anti-Stokes photons or exactly one anti-Stokes photon as the output of the source. The single-photon pulses are then stored and released by means of EIT techniques, i.e., the control field is turned off and after a delay time reapplied again. In experiment by Choi et al. the single-photon pulses are stored for $1 \mu \mathrm{s}$ in cold $\mathrm{Rb}$ atoms trapped in a magneto-optical trap (MOT) with overall transfer-storage-retrieval efficiency of $17 \%$. In other experiments by Chaneliere et al. and Eisaman et al. the overall efficiency was close to $6 \%$. The experiment by Choi et al. stands out because it exploits the entangled state of a photonic qubit. A single-photon from the atomic source is split on a beam splitter so that the two components of the input state of the form $\left|\psi_{i n}\right\rangle=1 / \sqrt{2}\left(|0\rangle_{A}|1\rangle_{B}+e^{i \varphi}|1\rangle_{A}|0\rangle_{B}\right)$ are directed into two atomic vapors. $[2,31]$ The EIT procedure is repeated now for two atomic ensembles. Subsequent tomography of the retrieved state verifies that the quantum memory conserved entanglement. The overall efficiency of transfer-storage-retrieval of entanglement is $20 \%$. The described experiments are proof-of-principle experiments rather than reliable implementations of quantum memories. Still they demonstrate significant progress. Naturally, for applications in a distributed quantum network the overall efficiency has to be much higher. The difficulty of relatively low efficiency of transfer-storage-retrieval process can be circumvented with an exploitation of atomic medium with increased optical depth $d$ and optimizing the shape of the control field with respect to the signal field. The efficiency of the optical quantum memory depends mostly on the optical depth $d$. However, 
the retrieval efficiency can be sharply increased if one uses the control field that stores the given signal field in optimal way.[2] In a recent experiment Novikova et al. used the iterative optimization procedure that maximized the storage and retrieval efficiency. First an initial optical pulse was stored and retrieved. Then time-reversed profile of the retrieved pulse was used as next input for the atomic memory. The whole procedure was repeated and converged very quickly to the optimal input pulse profile. The overall efficiency of the transfer-storage-retrieval process for optimal input field was close to $45 \%$. Moreover this experiment was performed for warm ${ }^{87} \mathrm{Rb}$ vapor with relatively low optical depth $d \simeq 9$. The exploitation of cold atomic vapors with increased optical depths should boost the overall lightstorage efficiency. The experiment of Novikova et al. confirmed again that the optical depth is the key figure of merit for the efficiency of quantum memories.

Finally, we would like to mention a quite interesting application of EIT technique, namely the possibility of building atomic-vapor-based high efficiency photon detectors with an estimated detection efficiency of $\eta_{D} \approx$ 99.8\%.[32] The single photons stored inside a medium can be counted by means of resonant fluorescence. Moreover, if the detection of light stored in an atomic ensemble does not alter the state of a medium, this kind of the detector could then realize a quantum nondemolition measurement of the photon-number operator since one can retrieve photons stored in atomic medium.[33]

Apart from EIT and Raman interaction, one may induce coherent behavior of a macroscopic atomic medium with a help of Rydberg atoms. In the following section we introduce a notion of the Rydberg state and the dipole-blockade mechanism.

\section{Rydberg state and dipole blockade mechanism}

Although the concept of Rydberg atom is known for more than 100 years, physicists are able to study them in laboratory only since nineteen seventies. Despite of this short period of experimental studies, we know already that Rydberg atoms allow for a number of interesting applications. The Rydberg state is a state of an alkali atom characterized by a high principal quantum number $n$.[34] Rydberg atoms possess a number of remarkable properties. To begin with, Rydberg atoms are very large compared to normal atoms. The radius of a Rydberg atom scales as $n^{2} a_{0}$, where $a_{0}$ is the Bohr radius, 
and the binding energy of a Rydberg state is given by

$$
E=-\frac{R}{(n-\delta)^{2}}=-\frac{R}{n^{*^{2}}}
$$

where $R$ is the Rydberg constant, $n^{*}$ is the effective quantum number, and $\delta$ is the quantum defect which corrects for the deviation from a case of the hydrogen atom.[35] This implies that the valence electron is very weakly bound to the nucleus. Moreover, the Rydberg states characterize with an incredibly long lifetime, which scales as $\tau_{0} n^{5}$ where $\tau_{0}$ is the typical lower level lifetime of around $\sim 10 \mathrm{~ns}$. Hence, Rydberg states possess lifetimes of order of ms and even longer.

Because of a very weak binding energy, the Rydberg atoms are extremely sensitivity to external electric fields. The Rydberg energy levels are easily perturbed by modest electric fields. Higher electric fields can even ionize Rydberg atoms. In fact, the ionization is commonly used as one of the detection methods. This sensitivity to electric fields is the source of a phenomenon called the dipole blockade mechanism. Atoms in Rydberg states have large dimensions and large dipole moments, resulting in a strong dipole-dipole interaction.[36] Under certain circumstances the effect of strong dipoledipole interaction can be observed in laboratory. The dipole blockade mechanism was observed experimentally in small clouds of alkali atoms, such as Rubidium in a vapor cell. [37, 38] This mechanism prevents populating states of an atomic ensembles with two or more atoms excited to the Rydberg level.[4] A single atom in a micron-sized atomic ensemble excited to a Rydberg state with a narrowband laser can inhibit excitation of the other atoms in the sample if the long range Rydberg-Rydberg interactions are much larger than a linewidth of the Rydberg state.

The physics of the dipole blockade mechanism is presented in Fig. 3. An optical pulse resonant with a transition to the Rydberg state $|r\rangle$ will create a Rydberg atom with a very large dipole moment [Fig. 3 (a.)]. For sufficiently short separations, the long range Rydberg-Rydberg interactions (dipole interactions) between the Rydberg atom and the other atoms will cause a shift in the Rydberg transition energy of the other atoms. Therefore, the optical pulse becomes off-resonant with the other atoms, and the ensemble is transparent to the pulse. Under dipole blockade conditions, the mesoscopic vapor behaves as one superatom with a two-level structure. A single excitation is coherently shared by all atoms in a sample and one is able to observe Rabi oscillations. Naturally, effectiveness of the blockade depends on an average strength of the interaction between atoms in the ensemble. 
(a.)

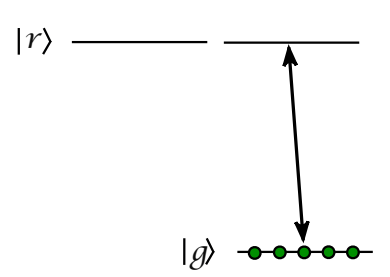

(6.)

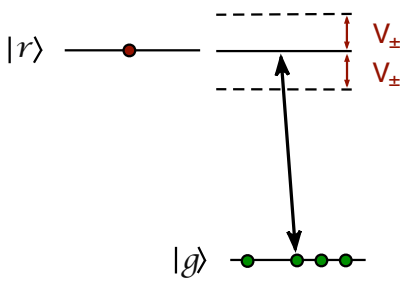

Figure 3: Diagram representing the dipole blockade mechanism. The ground state $|g\rangle$ and Rydberg state $|r\rangle$ are coupled by means of a narrowband laser. (a.) After an appropriate interaction time one of the atoms in atomic medium is excited to the Rydberg state $|r\rangle$. (b.) Presence of a single atom in the Rydberg state $|r\rangle$ shifts energy levels of all other atoms located within the long range Rydberg-Rydberg interactions distance and blocks any further excitations.

The long range Rydberg-Rydberg interactions have different type depending on the separation between atoms. The usual van der Waals interaction of types $C_{5} / R^{5}$ or $C_{6} / R^{6}$ can be resonantly enhanced by Förster processes to the $C_{3} / R^{3}$ long range interaction. In the absence of an external electric field, the Rydberg-Rydberg interactions are of the van der Waals type $C_{5} / R^{5}$ or $C_{6} / R^{6}$.[39] In a static electric field, a Rydberg atom possesses a large permanent dipole moment $p$, which scales as $\sim q a_{0} n^{2}$ with $q$ the electron charge, which leads to a much stronger and longer $C_{3} / R^{3}$ interaction. A pair of Rydberg atoms $i$ and $j$ interact with each other via dipole-dipole potential $V_{d d}$,

$$
V_{d d}=\frac{\mathbf{p}_{i} \mathbf{p}_{j}-3\left(\mathbf{p}_{i} \cdot \mathbf{e}_{i j}\right)\left(\mathbf{p}_{j} \cdot \mathbf{e}_{i j}\right)}{4 \pi \epsilon_{0}\left|\mathbf{r}_{i}-\mathbf{r}_{j}\right|^{3}}=\frac{p^{2}}{4 \pi \epsilon_{0} R^{3}}\left(1-3 \cos ^{2} \theta\right),
$$

where $\mathbf{e}_{i j}$ is a unit vector along the interatomic direction, $\theta$ is the angle between the interatomic separation $R=|\mathbf{R}|=\left|\mathbf{r}_{i}-\mathbf{r}_{j}\right|$ and the electric field $\mathbf{z}$ direction. In general, the interaction between Rydberg atoms can be quite strong. However, for some angles $V_{d d}$ vanishes which is undesirable for dipole blockade purpose.[39] Fortunately, there is another method to induce a strong, isotropic interaction between Rydberg atoms, comparable to $V_{d d}$. The resonant collisional process (Förster process) transfers energy between two atoms through the dipole-dipole interaction with strength $\rho_{1} \rho_{2} / R^{3}$, where $\rho_{1}$ and $\rho_{2}$ are the dipole matrix elements between initial and 
final energy states of the interacting atoms.[40] Therefore, the usual van der Waals interaction can be resonantly enhanced by Förster processes such as $n l+n l \rightarrow n^{\prime} l^{\prime}+n^{\prime \prime} l^{\prime \prime}$ when the $n l+n l$ states are degenerated in energy with the $n^{\prime} l^{\prime}+n^{\prime \prime} l^{\prime \prime}$ states. The Förster process induces an interaction potential of the form

$$
V_{ \pm}(R)=\frac{\delta}{2} \pm \sqrt{\frac{4 U_{3}(R)^{2}}{3}+\frac{\delta^{2}}{4}}
$$

where

$$
U_{3}(R)=q^{2}\left\langle n l\|r\| n^{\prime} l^{\prime}\right\rangle\left\langle n l\|r\| n^{\prime \prime} l^{\prime \prime}\right\rangle / R^{3},
$$

with $\delta=E\left(n^{\prime} l^{\prime}\right)+E\left(n^{\prime \prime} l^{\prime \prime}\right)-2 E(n l)$ is the Förster energy defect. There is no angular dependence for the potential $V_{ \pm}(R)$ so an interaction is isotropic. For perfect Förster degeneracy $(\delta=0) V_{+}(R)$ would be of similar strength and range to $V_{d d} \cdot[39]$ Although at the large separations, a non-zero Förster energy defect reduces long-range interaction between the atoms to be van der Waals $C_{6} / R^{6}$ type. However, if the Förster energy defects are smaller compared to the fine-structure splitting, then strong $C_{3} / R^{3}$ interaction can even occur at longer range.

Although Förster processes are very promising as a method to induce very long-range $C_{3} / R^{3}$ interactions, there are some selection rules that need to be fulfilled for obtaining high fidelity dipole blockade. Only for $l^{\prime}=l^{\prime \prime}=$ $l+1$ there are no so-called Förster zero states with $C_{3}=0$.[39] Therefore, a fidelity of the dipole blockade mechanism is highly dependent on a weakest interactions between degenerate Rydberg states and may be reduced under unfortunate circumstances. In the case of the Förster zero states, strength of the interaction between Rydberg atoms is not enhanced and reduces to the usual van der Waals long-range type. Therefore, a strong dipole blockade requires tuning of the resonances by means of an electric field.[39] The other possibility for attaining strong dipole blockade is to rely on the van der Waals interaction which at smaller distances, less than $5 \mu \mathrm{m}$, is large enough to mix the fine-structure levels together, so the interaction is of the $V_{d d}$ type.[41]

As one would expect, the concept of dipole blockade mechanism fueled a number of interesting proposals such as a method to entangle large numbers of atoms.[4] Fortunately, the exact strength of the dipole blockade in these proposals is not important as long as it is greater than the linewidth of a Rydberg state. Therefore, the atoms can be located at random distances $R$ from each other.[39] Moreover, with the dipole blockade mechanism at hand, one avoid a problem of mechanical interactions between atoms, since states with two or more atoms in the Rydberg state are never populated. Therefore, the atoms avoid heating and the internal states of the atoms are 
decoupled from the atomic motion.[4]

The range and quality of the dipole interaction has been studied extensively. In papers by Walker and Saffman the primary errors that enter the blockade process were analyzed.[38, 41] Naturally, the two most common errors are the occurrence of doubly-excited Rydberg states and singly-excited states outside the desired two-level system. In the case of Rubidium atoms with principal quantum number $n=70$, the blockade energy shift is approximately $1 \mathrm{MHz}$. Hence, a strong and reliable blockade is possible for two atoms with separation up to $\sim 10 \mu \mathrm{m}$.[41] Moreover, decoherence associated with spontaneous emission from long-lived Rydberg states can be quite low ( $\sim 1 \mathrm{~ms}$ ). The dipole blockade mechanism can be used to build fast quantum gates, i.e., a two qubit phase gate.[42, 43, 44] The long-range dipole-dipole interaction between atoms can be employed to realize a universal phase gate between pairs of single-photon pulses.[45, 46, 47] Most importantly, the ideas based on the dipole blockade mechanism are experimentally feasible.

The single quantum sensitivity suggests that the dipole blockade mechanism can be used to create cluster (graph) states: The blockade mechanism can be used in a heralding type of entangling operations and render them to be nearly deterministic.[48] Before introducing nearly deterministic entangling protocol based on the dipole blockade mechanism, let us first review several schemes for probabilistic entanglement generation between atomic vapors.

\section{Entanglement in atomic ensembles}

Initially, atomic vapors were proposed as fast quantum interfaces. However, it is also possible to define a qubit (stationary qubit or quantum processor) in an atomic ensemble, and the question remains how to implement the entangling operations between the qubits that enable universal distributed quantum computation. One may choose to create a large network of spatially separated quantum processors and connect them with quantum communication channels. However, in fact it suffices to create a inherently distributed, large entangled multi-qubit resource - the graph state - after which the entire computation proceeds via single-qubit measurements.[49, 50] Graph states are large arrays of isolated qubits connected (entangled) via CZ operations. The graph states are a scalable resource and can be built up with probabilistic entangling operations with $p_{\text {success }}>0$. [51] When the success probability of entangling operation is low, a very large overhead in optical elements is required. Moreover, finite coherence times of the qubits limit 
practical use of the graph states. Hence, it is extremely important to build them up in an efficient way.

In this section, we are going to focus on the probabilistic entanglement generation between two distant qubits implemented as atomic ensembles. We are especially interested in the heralded entanglement generation, i.e., detection of an object such as single photon heralds the creation of entanglement between two distant macroscopic objects such as atomic ensembles. [52] The heralded protocols work with some success probability $p_{\text {success }}$, which in principle depend on structure of the protocol, the efficiency of detection method and the physical implementation. Therefore, for efficient entanglement generation one has two choices: either prepare many copies of physical systems or repeat entanglement procedure sufficient number of times. If $p_{\text {success }}$ is small, it takes on average $1 / p_{\text {success }}$ copies or repetitions to create entanglement between two distant ensembles on average. The probabilistic nature of the heralded entanglement procedures imposes some limitations on its practical use in quantum computation but not in quantum communication.

\subsection{The DLCZ protocol}

One of the well known entangling protocols is the DLCZ protocol. It was devised by Duan et al. as a quantum repeater protocol. The quantum repeaters are essential for long distance quantum communication. The DLCZ protocol is realized on two macroscopic atomic ensembles, balanced beam splitter and two single-photon photodetectors. The relevant atomic level structure is shown in Fig. 4. The $N$ atoms in an ensemble have two lower, long lived energy states $|g\rangle,|s\rangle$ (Zeeman sublevels of the ground state), and an excited state $|e\rangle$. The protocol begins with all atoms prepared in the ground state $|g\rangle$. Then a weak laser pulse that addresses off-resonantly $|g\rangle$ $|e\rangle$ transition transferees, preferably a single atom to the state $|s\rangle$ and simultaneously produces a single, forward-scattered Stokes photon. This process resembles stimulated Raman passage (STIRAP) and the whole state of the ensemble-light system is given by

$$
|\phi\rangle_{E L}=|0\rangle_{E}|0\rangle_{L}+\sqrt{p_{e}}|S\rangle_{E}|1\rangle_{L}+\mathcal{O}\left(p_{e}\right)
$$

where $|0\rangle_{E}$ is the ensemble collective ground state given by $|0\rangle_{E}=\left|g_{1}, g_{2}, \ldots, g_{N}\right\rangle$, $|0\rangle_{L}$ is the vacuum state of light, $|S\rangle_{E}$ is the collective state of the ensemble given by $|S\rangle_{E}=1 / \sqrt{N} \sum_{j=1}^{N}\left|g_{1}, g_{2}, \ldots, s_{j}, \ldots, g_{N}\right\rangle,|1\rangle_{L}$ represents the single forward-scattered Stokes photon state and $p_{e}$ is the excitation probability that because of weak excitation laser pulse is small. The above state 
represents the very heart of the DLCZ protocol. The STIRAP procedure can be applied simultaneously to two ensembles. In the result, a single forwardscatted Stokes photon is produced in one of the ensembles. It is not possible to learn which ensemble is a source of a Stokes photon. The light modes from both ensembles are then combined on the balanced beam splitter (BS) to erase which-path information (see Fig. 4 (a.)). Following the detector click on one of the photodetectors $\left(\mathrm{D}_{1}, \mathrm{D}_{2}\right)$ the maximally entangle state of two ensembles $\Psi_{ \pm}=1 / \sqrt{2}\left(|S\rangle_{A}|0\rangle_{B} \pm|0\rangle_{A}|S\rangle_{B}\right)$ is created. This scheme works with the probability given by $p_{e}$. Hence, the entangled state will be generated on average after $1 / p_{\text {success }}$ procedure repetitions. As mentioned above,
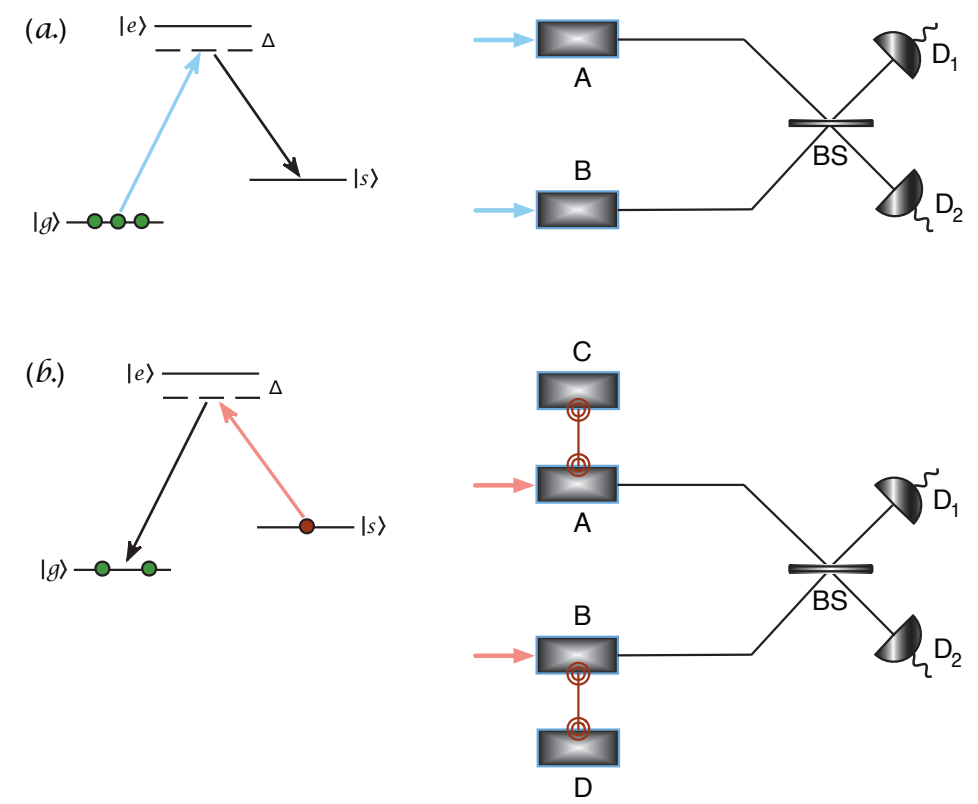

Figure 4: (a.) The relevant three-level $\Lambda$-type structure and diagram of the DLCZ protocol. $|g\rangle$ and $|s\rangle$ are a lower, long lived energy states and $|e\rangle$ is an excited state. The blue line represents a weak, write laser pulse. Conditionally on the detector click entanglement is created between A-B ensembles. (b.) Diagram of the entanglement swapping procedure. The red line represents a read-out laser pulse. Conditionally on the detector click entanglement is extended to C-D ensembles.

the DLCZ protocol is in fact a quantum repeater protocol. The DLCZ protocol enables the entanglement of two atomic ensembles and then through 
swapping entanglement, the connection can be established between distant sites.[1] In other words, the DLCZ protocol enables the distribution of entanglement between distributed quantum network nodes. If one prepares two pairs of atomic ensembles (A-C and B-D) in the maximally entangled state, then by means of a read-out laser pulse, applied to the $|s\rangle-|e\rangle$ transition, stored atomic excitation of a single ensemble in each pair can be converted into light modes (see Fig. 4 (b.)). These light modes are again combined on the balanced beam splitter to erase which-path information, and, conditionally on the detector click, entanglement is extended to the more distant C-D ensembles. This procedure is called an entanglement swapping, and can in principle be applied many times creating a communication channel between distant nodes.

The interesting feature of the DLCZ protocol is the fact that it has built-in entanglement purification. The fidelity imperfection of the protocol is proportional to the $p_{e}$ and can be lowered close to zero for small excitation probabilities.[1] The DLCZ protocol is scalable and highly efficient in comparison with direct communication methods. Apart from the communication applications, the DLCZ protocol can be used for quantum teleportation, cryptography and to show the violation of a Bell inequality.

The DLCZ protocol drew a lot of attention from experimental groups all around the world. The first experimental attempts to realize the quantum repeater were limited to the generation of nonclassical photon pairs originating from a single atomic ensemble. $[25,26,53]$ In these experiments by means of a write pulse (Raman adiabatic passage) a collective atomic state is created together with a single Stokes photon. After some programmable delay time, the read pulse is applied to the atomic ensemble resulting in a generation of second photon - anti-Stokes photon. The quantum (nonclassical) character of correlations between both photons is confirmed by the violation of a Cauchy-Schwarz inequality. [25, 26, 53] Although none of the mentioned experiments implemented the DLCZ protocol, techniques used in these experiments are considered as a first and a crucial step in the realization of the protocol.[25] Shortly after these initial experiments, the full quantum repeater implementations were realized.[27, 31, 54, 55, 56] The DLCZ protocol was realized on the atomic ensembles consisting of $\sim 10^{5}$ atoms separated by few meters.[54] The experiments involve preparation of the collective atomic states and the read out of quantum memories after some delay time. The quality of the entanglement between quantum nodes is given in terms of concurrence $C[27,31,54]$ or validated by the violation of the Bell inequality. $[55,56]$ There are several factors that limit the performance of DLCZ protocol. The main one is low retrieval efficiency varying in 
range from $30 \%$ to $60 \%$ and decoherence of the collective atomic states.[27]

An interesting extension of the quantum repeater protocol to include quantum teleportation was devised by Chen et al. They demonstrated teleportation between photonic and atomic qubits.[57] The quantum state of a single photon was teleported onto an atomic ensemble, stored for up to $8 \mu$ s and then converted back to a photonic state.[57] The main advantage of this scheme over other teleportation protocols is the prospect of storing the teleported state and reusing it for further quantum applications. Although this technique makes large-scale communication and distributed quantum computation more feasible, it is not yet useful for practical applications due to many experimental limitation, such as short quantum memory lifetime.

\subsection{The double-heralding protocol}

Another protocol for probabilistic entanglement generation between spatially separated quantum nodes is the double-heralding protocol. Here, entanglement is established after two consecutive single photon detections, hence the name of the protocol. The double-heralding protocol is based on matter qubits and linear optics. Let us consider a two spatially separated matter qubits, e.g. single atoms or atomic ensembles, each having two lower energy levels $|g\rangle$ and $|s\rangle$, and an excited level $|e\rangle$, which is coupled only to the $|g\rangle$ level by means of an optical pulse. [51, 58] If a matter qubit is realized by an atomic ensemble, the above energy levels are represented by collective atomic states. The energy levels $|g\rangle$ and $|s\rangle$ constitute the qubit states. The protocol begins with both matter qubits prepared in the separable state $|\phi\rangle_{L R}=\frac{1}{2}(|s\rangle+|g\rangle)_{L}(|s\rangle+|g\rangle)_{R}$. We apply an optical $\pi$ pulse to each qubit which results in a single photon being emitted when a matter system spontaneously goes back to the $|g\rangle$ level. Following above manipulations, the total state of the matter qubits and output modes of light is given by

$$
|\Phi\rangle=\frac{1}{2}(|s s\rangle|00\rangle+|s g\rangle|01\rangle+|g s\rangle|10\rangle+|g g\rangle|11\rangle),
$$

where $|0\rangle$ and $|1\rangle$ denote the vacuum and a single photon state, respectively. The modes of light are then combined on the balanced beam splitter (BS) to erase which-path information, which results in the state:

$|\Phi\rangle=\frac{1}{2}\left\{|s s\rangle|00\rangle+\frac{1}{\sqrt{2}}[(|s g\rangle+|g s\rangle)|01\rangle+(|s g\rangle-|g s\rangle)|10\rangle+|g g\rangle(|20\rangle+|02\rangle)]\right\}$.

Following the beam splitter, the light modes are coupled to the regular photodetectors that must have a low dark count rate. Conditional on a single 
detector click $D_{ \pm}$the state of the matter qubits is given by the following density operator:

$$
\rho^{( \pm)}=\frac{1}{2-\eta}\left|\Psi^{ \pm}\right\rangle\left\langle\Psi^{ \pm}\left|+\frac{1-\eta}{2-\eta}\right| g g\right\rangle\langle g g|,
$$

where $\left|\Psi^{ \pm}\right\rangle=\frac{1}{\sqrt{2}}(|s g\rangle \pm|g s\rangle)$ and $\eta$ is the combined photon collection and detection efficiency.[51] The above state is a mixed state. To remove the second, separable part of the mixed state $\rho^{( \pm)}$, a bit flip must be applied to both matter qubits. In the case of a matter qubit implemented as atomic ensemble, a bit flip is not a trivial operation. In fact, for reliable bit flip operation one has to make use of the dipole-blockade mechanism. In the next section, we review the concept of an atomic ensemble as single qubit system and analyze in detail a scheme for single-qubit operations in atomic ensembles. After a bit flip, we repeat the whole procedure. Therefore, after a second measurement event (single detector click in $D_{ \pm}$) the total state of two qubits is projected onto the pure maximally entangled state

$$
\left|\Psi^{ \pm}\right\rangle=\frac{1}{\sqrt{2}}(|s g\rangle \pm|g s\rangle)
$$

with success probability $p=\frac{\eta^{2}}{2}$ and unit fidelity. The double-heralding protocol can be used to efficiently create multi-qubit graph states with only moderate overhead in physical resources, which together with the oneway model of computation can be used to implement universal quantum computation. [51, 59] The procedure is a fully scalable scheme for universal quantum computation assuming that the physical implementation allows high-fidelity single qubit operations and measurements.

The double-heralding protocol posses many attractive features. The scheme is based on the simple level structure and simple optical network which imply rather straightforward phase stabilization. Moreover, the protocol works for distributed qubits which facilitate control of decoherence and permits applications in quantum communication such as quantum repeaters. The main disadvantage of the double-heralding protocol is the success probability $\left(p=\frac{\eta^{2}}{2}\right)$ depending on the collection of photon which makes it sensible to photon loss. This problem was addressed by the broker-client model devised by Benjamin et al.[60] In the broker-client model two qubits are placed in each node. One of them is used for entanglement generation between nodes and the other one serves for storing of the entanglement when double-heralding procedure succeeds. In this way, influence of a extreme photon loss is suppressed and effective graph state generation with small overhead in physical resources is feasible. 
From the experimental point of view one of the main challenges in implementing double-heralding protocol is generation of the indistinguishable photons. However, the following experiments prove that this is possible. There is a number of physical systems that may be used to represent a matter qubit such as trapped ions or atoms, NV centers in diamond and Pauli blockade quantum dots.[58] In the experiment by D. L. Moehring et al.[61] two trapped ${ }^{171} \mathrm{Yb}^{+}$ions are separated by one meter. Each of the ions emits a single photon which polarization is entangled with each ion. The single photons are then combined on the balanced beam splitter and detected by photon-counting photomultiplier tubes (PTMs). In this experiment entanglement is generated for the system with more complex level structure. However, in the essence the mentioned experiment and the double-heralding protocol are analogous. The entanglement between ions is confirmed by violation of a Bell inequality.[62] Unfortunately when all experimental limitations are taken into the account heralded entanglement between ions is established every $8.5 \mathrm{~min}$. This result is consistent with a general observation that for $\eta \ll 1$ the success probability of the generation of a maximally entangled state of even two qubits can be quite low. Therefore, generation of multi-qubit graph states for quantum computation, or quantum communication has to be based on protocols with higher success probabilities.

A deterministic protocol for implementing a universal two-qubit gate between two atoms placed in optical cavities was proposed by Lim et al. [63] The two-qubit interactions are induced using single-photons, that originate from atom-cavity matter systems, linear optics and photodetectors. The qubits are encoded in two atomic ground states and prepared in an arbitrary state $|\Psi\rangle=\alpha|0\rangle+\beta|1\rangle$. Subsequently, an encoding operation is applied to each matter system that transforms the state of each qubit to $|\Psi\rangle=\alpha|0, E\rangle+\beta|1, L\rangle$. In other words, each atom in an optical cavity emits a single photon at an early (E) or a late (L) time. The atom-cavity system acts as "on-demand" single photon source. Therefore, the encoded state contains both the initial state of an atom-cavity matter system and the state of single photon. This is so-called time-bin encoding. Consequently, one can prepare two atom-cavity systems and two single photons in the arbitrary encoded state. At this point, the measurement in the appropriate basis (mutually unbiased basis formed by single photon basis states $|E\rangle$ and $|L\rangle)$ can realize any universal two-qubit gate.[64] However, some certain experimental implementations and measurement bases may involve rather complicated linear optical setups. The main strength of this protocol is its repeatability. If the proposed gate fails then the original input state can be 
recovered by local operations and whole procedure can be repeated until successful operation is achieved.[64] On average two repetitions are required to realize the gate operation. This repeat-until-success modus operandi leads to the deterministic two-qubit gate operations. Unfortunately, in the presence of unavoidable photon loss, the above procedure becomes probabilistic. [64] The successful implementation of the two-qubit gates requires detection of a photon pair in appropriate outputs of optical network and the failure associated with photon emission, collection or detection leaves the matter qubits in an unknown state. Although, the failure of the scheme is heralded and scalable quantum computing is still possible, the overall overhead cost associated with the procedure may be increased significantly.[64]

Here, we present another entangling protocol that in principle is also deterministic. This protocol employs dipole blockade mechanism between Rydberg atoms. We show how to efficiently create graph states using single photons interacting with atomic ensembles via the dipole blockade mechanism. The protocol requires identical single-photon sources, one atomic medium per physical qubit placed in the arms of a Mach-Zehnder interferometer, and regular photodetectors. We present a general entangling procedure, as well as a procedure that generates $Q$-qubit GHZ states with success probability $p_{\text {success }} \sim \eta^{Q / 2}$, where $\eta$ is the combined detection and source efficiency. This is significantly more efficient than any known robust probabilistic entangling operation. $[64,51]$ The GHZ states are locally equivalent to the graph state and form the basic building block for universal graph states. Our protocol significantly reduces an overhead in optical elements and leads to better quantum computing prospects. However, before giving a detailed description of a new entangling operation, let us review a scheme for implementing single-qubit operations on the qubit defined in atomic ensemble and analyze it in detail.

\section{Atomic ensemble as single qubit system and single- qubit gates}

Until now we have avoided the issue of single qubit operations in atomic media. An atomic ensemble can serve as a qubit as long as one is able to apply single qubit rotations. A qubit may be represented by a micron-sized atomic ensemble, cooled to $\mu \mathrm{K}$ temperatures by the far off-resonant optical trap (FORT) or magneto-optical trap (MOT). The $N$ atoms at positions $r_{j}$ in an ensemble have three lower, long lived energy states $|g\rangle,|e\rangle$, and $|s\rangle$ (see Fig. 5). The qubit states in a mesoscopic ensemble are collective states 
are defined as

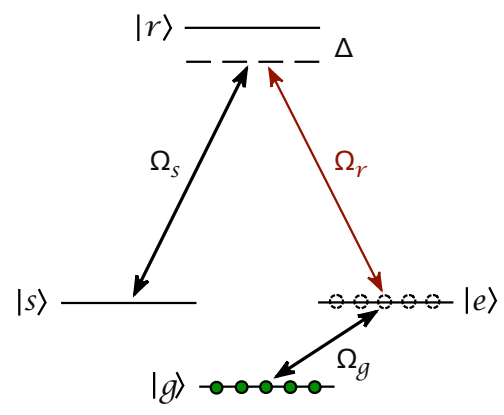

Figure 5: Diagram of atomic level structure with allowed atomic transitions. States $|g\rangle,|e\rangle$, and $|s\rangle$ can be realized by a lower, long lived energy states of alkali atoms. $\Omega_{g}$ denotes Rabi frequency of a laser pulse coupling $|g\rangle$ and $|e\rangle$ states. A second laser pulse $\Omega_{s}$ is applied to the transition between the highly excited Rydberg level $|r\rangle$ and the state $|s\rangle$. This transition may possibly be a two-photon process. $\Omega_{r}$ denotes Rabi frequency of a weak laser pulse coupling $|e\rangle$ and $|r\rangle$ states. Both laser pulses $\Omega_{r}$ and $\Omega_{s}$ may be detunned from the corresponding atomic transition by $\Delta$.

$$
\begin{aligned}
|0\rangle_{L} & \equiv|g\rangle=\left|g_{1}, g_{2}, \ldots, g_{N}\right\rangle \\
|1\rangle_{L} & \equiv|s\rangle=\frac{1}{\sqrt{N}} \sum_{j=1}^{N} e^{i \mathbf{k} \cdot \mathbf{r}_{j}}\left|g_{1}, g_{2}, \ldots, s_{j}, \ldots, g_{N}\right\rangle .
\end{aligned}
$$

Energy levels $|g\rangle$ and $|s\rangle$ play the role of storage states and transition between these states is always dipole-forbidden. These qubits states have a very desirable property of long coherence times. However, in the case of the qubit states defined as collective states of mesoscopic ensemble, the singlequbit manipulations are more complex than in the case of a qubit realized on a single atom. Moreover, one cannot use the weak excitation regime to implement reliable single-qubit operations. In fact, the simplest approach to this problem is to realize single-qubit rotations by means of classical optical pulses and the dipole blockade mechanism. In a paper by Brion, Mølmer, and Saffman[44], the single-qubit rotations are performed with only three laser pulses (see Fig. 6). The laser pulses illuminate the entire ensemble and excite all atoms with equal probability. [4] The states $|e\rangle$ and $|r\rangle$ participate in the interaction part of the scheme. First, a $\pi$ pulse transfers a single atom from $|s\rangle$ to $|r\rangle$, then a coherent coupling of states with zero and one 
Rydberg excited atom is applied for appropriate amount of time and finally, a $\pi$ pulse transfers a single atom from $|r\rangle$ to $|s\rangle$ (the reader may notice that two additional $\pi$ pulses are depicted in Fig. 6 that transfer population from the storage level $|g\rangle$ to $|e\rangle$ and back again). Therefore, in the case of a bit flip operation $(X)$ the coherent coupling is just a $\pi$ pulse with a real Rabi frequency, and the Hadamard gate $(H)$ can be performed by a $\pi / 2$-pulse on the same transition. An arbitrary phase gate $\Phi(\phi)=\exp (-i \phi Z / 2)$ is realized by a detuned optical pulse applied to the transition between $|s\rangle$ and an auxiliary level $|a\rangle$ (not shown in Fig. 6). The gates $\Phi(\phi), X$, and $H$ generate all single-qubit operations. The readout of a qubit is based on the resonance fluorescence and again requires an auxiliary level $|a\rangle$. An optical laser drives a transition between $|s\rangle$ and $|a\rangle$ producing a large number of fluorescence photons. If the measurement gives no fluorescence photons, the qubit is in $|0\rangle_{L}$. Otherwise, a state of the qubit is projected into $|1\rangle_{L}$.

In summary, all single-qubit manipulations can be implemented in the dipole blockade regime with a laser pulse of a well-defined length and phase resonant with transition between a lower energy level and Rydberg state. The single-qubit manipulations can be executed rather fast through collective enhancement. The collective enhancement emerge from the fact that although only one atom is excited to Rydberg state, all $N$ atoms in atomic medium interact with a laser field. In general, the above technique for implementing single-qubit manipulation is capable of generating any superpositions of collective qubit states of mesoscopic ensembles.

(a.)

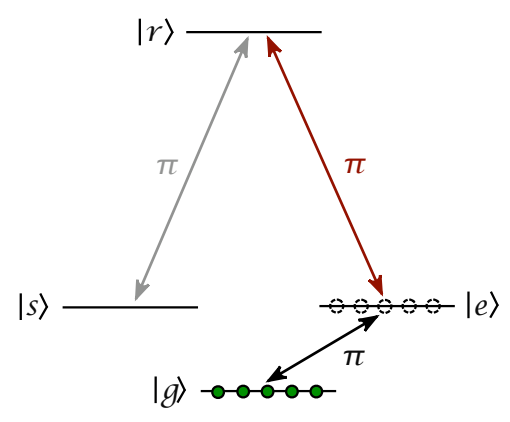

(6.)

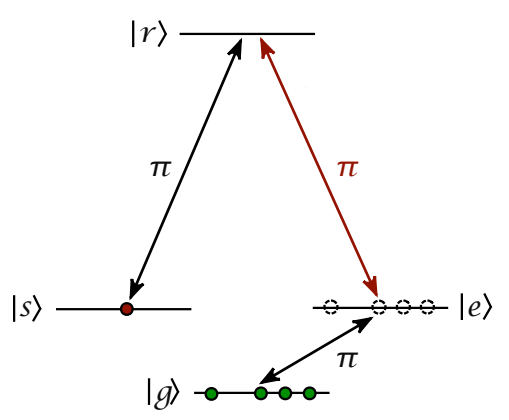

Figure 6: Diagram representing the bit flip operation $(X)$. (a.) Rotation from $|0\rangle_{L}$ to $|1\rangle_{L}$ and (b.) rotation from $|1\rangle_{L}$ to $|0\rangle_{L}$. See the text for an explanation. 
The scheme for implementing single-qubit operation relies heavily on the dipole blockade mechanism. We analyze the above scheme for the case of a bit flip operation $X$. Therefore, we need to carefully consider the evolution of the system under a $\pi$ pulse applied to the transition between $|e\rangle$ and $|r\rangle$. In the following discussion, we use levels $|g\rangle$ and $|e\rangle$ to denote a lowlying level. In general, the interaction of atoms with an optical laser pulse,

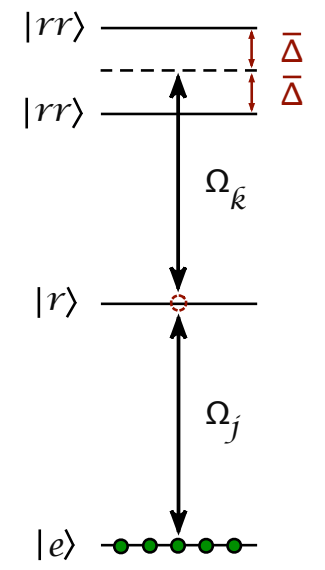

Figure 7: The dipole blockade mechanism. The level structure consists of collective states of a mesoscopic atomic ensemble. The state $|e\rangle$ is the collective low-lying state, $|r\rangle$ is the singly excited Rydberg state and $|r r\rangle$ is the doubly excited state. $\Omega$ is the Rabi frequency of a weak laser pulse that is out of resonance with the transition between single and double excited states. $\bar{\Delta}$ is the mean dipole shift induced by a presence of a single atom in Rydberg state.

within the dipole approximation and in the rotating frame approximation, is governed by interaction Hamiltonian $\hat{H}_{\text {int }}$

$$
\begin{aligned}
\hat{H}_{i n t}= & -i \hbar \sum_{j=1}^{N} \Omega_{j} \sigma_{r e}^{j} \exp \left[i\left(\omega_{r e}-\omega\right) t\right] \\
& -i \hbar \sum_{j, k>j}^{N} \Omega_{k} \sigma_{r r}^{j k} \exp \left[i\left(\omega_{r e}-\omega\right) t\right]+\text { H.c. }
\end{aligned}
$$

where $\Omega_{j}=\Omega e^{i \mathbf{k} \cdot \mathbf{r}_{j}}$ is the Rabi frequency, $\omega=k c$ is the frequency of an optical laser pulse, $\sigma_{r e}^{j}=\left|r_{j}\right\rangle\langle e|$ and $\sigma_{r r}^{j k}=\left|r_{j} r_{k}\right\rangle\left\langle r_{j}\right|$ are the atomic transition 
operators (see Fig. 7).[65] The first transition operator $\sigma_{r e}^{j}$ corresponds to the transition between the collective state $|e\rangle$ and the singly excited state

$$
|r\rangle=\frac{1}{\sqrt{N}} \sum_{j=1}^{N} e^{i \mathbf{k} \cdot \mathbf{r}_{j}}\left|r_{j}\right\rangle
$$

where $\left|r_{j}\right\rangle=\left|e_{1}, e_{2}, \ldots, r_{j}, \ldots, e_{N}\right\rangle$. The second one corresponds to the transition between the singly excited state $|r\rangle$ and the doubly excited state

$$
|r r\rangle=\sqrt{\frac{2}{N(N-1)}} \sum_{j, k>j}^{N} e^{i\left(\mathbf{k} \cdot \mathbf{r}_{j}+\mathbf{k} \cdot \mathbf{r}_{k}\right)}\left|r_{j} r_{k}\right\rangle,
$$

where $\left|r_{j} r_{k}\right\rangle=\left|e_{1}, e_{2}, \ldots, r_{j}, \ldots, r_{k}, \ldots, e_{N}\right\rangle$. We assume that the optical laser pulse is resonant with a transition between $|e\rangle$ and $|r\rangle\left(\omega_{r e}-\omega=0\right)$. Then, the dipole interaction between two Rydberg atoms is given by

$$
\hat{V}_{d d}=\hbar \sum_{j, k>j}^{N} \Delta_{j k}\left|r_{j} r_{k}\right\rangle\left\langle r_{j} r_{k}\right|
$$

where $\Delta_{j k}=\frac{C_{6}}{\left|\mathbf{r}_{j}-\mathbf{r}_{k}\right|^{6}}$ is the dipole shift of the weakest van der Waals type. Hence, the coupling of levels $|e\rangle$ and $|r\rangle$ is described by the Hamiltonian $\hat{H}=\hat{H}_{i n t}+\hat{V}_{d d}$. The state vector of an atomic ensemble is given by

$$
|\psi(t)\rangle=c_{g}|g\rangle+\sum_{j=1}^{N} c_{j} e^{i \mathbf{k} \cdot \mathbf{r}_{j}}\left|r_{j}\right\rangle+\sum_{j, k>j}^{N} c_{j k} e^{i\left(\mathbf{k} \cdot \mathbf{r}_{j}+\mathbf{k} \cdot \mathbf{r}_{k}\right)}\left|r_{j} r_{k}\right\rangle .
$$

In the limit where the dipole shift is much larger than the Rabi frequency of an optical laser pulse $\Delta_{j k} \gg \Omega_{j}$, the Schrödinger equation for amplitudes of the state vector gives

$$
\begin{aligned}
\dot{c}_{g} & =\sqrt{N} \Omega c_{r} \\
\dot{c}_{r} & =-\sqrt{N} \Omega c_{g}+\frac{\Omega}{\sqrt{N}} \sum_{j, k>j}^{N} c_{j k} \\
\sum_{j, k>j}^{N} \dot{c}_{j k} & =-\sum_{j, k>j}^{N} \Omega c_{j}-i \sum_{j, k>j}^{N} c_{j k} \Delta_{j k},
\end{aligned}
$$

with $c_{r}=\sqrt{N} c_{j}$. [65] Elimination of the doubly excited Rydberg state described by Eq. $(24)$ by means of an adiabatic approximation $\left(\dot{c}_{j k} \approx 0\right)$ 
yields

$$
\begin{aligned}
\dot{c}_{g} & =\sqrt{N} \Omega c_{r}, \\
\dot{c}_{r} & =-\sqrt{N} \Omega c_{g}+\frac{i \bar{\Delta} \Omega^{2}}{N} c_{r},
\end{aligned}
$$

where $\bar{\Delta}=\sum_{j, k>j}^{N} \frac{1}{\Delta_{j k}}$ is the mean dipole shift. The solution of Eq. (26) for $c_{g}(0)=1$ (initially all atoms are in a low-lying state $|g\rangle$ ) reads as

$$
\left|c_{r}(t)\right|^{2}=\sin ^{2}(\sqrt{N l} \Omega t) / l,
$$

with $l=1+\frac{\bar{\Delta}^{2} \Omega^{2}}{4 N^{3}}$. The evolution from collective state $|e\rangle$ to singly excited state $|r\rangle$ in time $t=\frac{\pi}{2 \sqrt{N l} \Omega}$ occurs with probability $P_{1}=1 / l$. In the limit of finite dipole blockade, the probability of unwanted double excitations after the $\pi$ pulse is given by

$$
P_{2}=\sum_{j, k>j}^{N}\left|c_{j k}\right|^{2}=\frac{\bar{\Delta}_{P_{2}} \Omega^{2}}{N},
$$

with $\bar{\Delta}_{P_{2}}=\sum_{j, k>j}^{N} \frac{1}{\Delta_{j k}^{2}}$. A finite blockade also implies a frequency shift of the effective two-level system $(|e\rangle$ and $|r\rangle)$. The resonance frequency is shifted by $\delta \omega=\Omega^{2} \bar{\Delta} / N$. The above results can be applied to the case of any single-qubit operation.

The numerical values based on the above model for single-qubit rotation are obtained for the following situation. We assume that a qubit is realized by a quasi one-dimensional (cigar shaped) atomic vapor consisting of $\sim 500$ ${ }^{87} \mathrm{Rb}$ atoms. The spatial distribution (probability density) of an atomic cloud is given by

$$
P(z)=\left(2 \pi \sigma_{z}^{2}\right)^{-1 / 2} \exp \left(-z^{2} / 2 \sigma_{z}^{2}\right)
$$

where $z$ is a dimension along the ensemble, $\sigma_{z}=3.0 \mu \mathrm{m}$ is the variance in $z$ direction and $\sigma_{x y}=0.5 \mu \mathrm{m}$ is the variance in transverse directions. The level $|r\rangle$ may correspond to $43 D_{5 / 2}$ or $58 D_{3 / 2}$ state. The probability of double excitation given by Eq. (28) can be rewritten in terms of the mean blockade shift $B$ with $1 / B^{2}=2 \bar{\Delta}_{P_{2}} / N(N-1)$.[41] Hence, the probability of double excitation is given by

$$
P_{2}=\frac{\Omega_{N}^{2}(N-1)}{2 N B^{2}}
$$


where $\Omega_{N}=\sqrt{N} \Omega$. For $43 D_{5 / 2}$ and $58 D_{3 / 2}$ state, the mean blockade shift is $B=2 \pi 0.25 \mathrm{MHz}$ and $B=2 \pi 2.9 \mathrm{MHz}$ in a trap with $\sigma=3.0 \mu \mathrm{m}$, respectively.[41] For $\Omega=2 \pi 1 \mathrm{kHz}$, the probability of double excitation for the $43 D_{5 / 2}$ level is $P_{2} \cong 4.010^{-3}$ and for the $58 D_{3 / 2}$ level is $P_{2} \cong 3.010^{-5}$. The probability of doubly-excited states and singly-excited states outside the desired two-level system resulting from a shifted resonance frequency are similar.

The time of a $\pi$ pulse applied to the transition between $|e\rangle$ and $|r\rangle$ is $t \cong 11.2$ $\mu \mathrm{s}$. We estimate that the rest of the $\pi$ pulses which are necessary to realize any single-qubit rotation (see Fig. 6) can be applied in time significantly shorter than the time $t$. In summary, above experimental implementation of the single-qubit rotations can be carried out on a microsecond timescale. The spontaneous emission from the Rydberg state and the black-body transfer (to other Rydberg states) occur with low rates of order $10^{3} \mathrm{~Hz}$ (or even $10^{2} \mathrm{~Hz}$ for higher Rydberg states) and may introduce small error $P_{\text {decay }} \cong 1-\exp \left(-10^{3} t\right)=0.01$.[66] Other sources of errors such as atomic collisions and Doppler broadening are negligible because of a low temperature of the atomic vapor.

The fidelity of the single-qubit rotations can be as high as $F_{\text {single }}=$ $\exp \left[-\left(2 P_{2}+P_{\text {decay }}\right)\right] \cong 0.99$, where $P_{2}=3.010^{-5}$. This fidelity is given for the worst case scenario when the separation of atoms is maximal and the dipole-dipole interaction is of the weakest (van der Waals) type.

The single-qubit rotations are one of the basic operations that are necessary in any model of quantum computation. The above fast and reliable implementations of the single-qubit operations open a possibility for a realization of the measurement-based model of quantum computation. However, we are still lacking a scheme for efficient generation of the cluster states, a resource for the one-way quantum computer.

\section{New entangling protocol based on the dipole blockade mechanism}

We propose a scheme for efficient and reliable cluster state generation, based on the dipole blockade mechanism.[48] The entangling operation between two mesoscopic atomic ensembles takes place in the arms of a Mach-Zehnder interferometer shown in Fig. 8. The protocol begins with both ensembles $A$ and $B$ prepared in the collective state $|\phi\rangle_{A, B}=|e\rangle \equiv\left|e_{1}, e_{2}, \ldots, e_{N}\right\rangle$ (see Fig. 5). Next, two indistinguishable photons enter each input mode of the interferometer. After the first beam splitter $\left(B S_{1}\right)$, due to the Hong-Ou- 


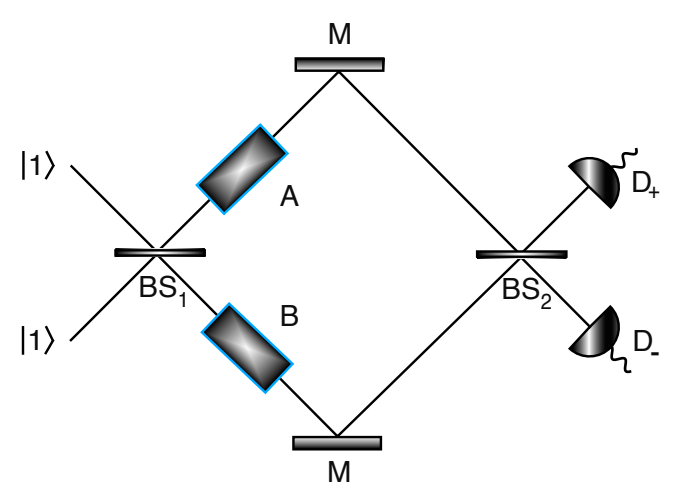

Figure 8: Diagram of the entangling protocol. A pair of entangled photons in the state $|\phi\rangle_{\text {light }}=\frac{i}{\sqrt{2}}(|02\rangle+|20\rangle)$ interact with atomic vapors placed in the arms of a Mach-Zehnder interferometer. One and only one alkali atom in the ensemble is excited by one of the photons to the Rydberg state $|r\rangle$. Absorption of the second photon is prohibited by the dipole blockade mechanism. Detection of a single photon will leave the atomic ensembles in an entangled state $\left|\psi^{ \pm}\right\rangle=\frac{1}{\sqrt{2}}(|r e\rangle \pm i|e r\rangle)$.

Mandel (HOM) effect two photons propagate in the maximally entangled state:

$$
|\phi\rangle_{\text {light }}=|11\rangle \stackrel{B S_{1}}{\longrightarrow} \frac{i}{\sqrt{2}}(|02\rangle+|20\rangle),
$$

where $|0\rangle$ and $|2\rangle$ denote the vacuum and a two-photon state, respectively. [67, 68] The photons can be entangled only if prior to a beam splitter interaction both photons were exactly the same in all possible senses. Subsequently, two photons interact with the atomic ensembles: One and only one atom in the ensemble is excited by one of the photons to the Rydberg state $|r\rangle$, and the absorption of the second photon is prohibited by the dipole blockade mechanism.[48] Following the dipole blockade interaction, the total state of atomic ensembles and light fields is given by

$$
|\phi\rangle_{i n t}=\frac{i}{\sqrt{2}}(|e r\rangle|01\rangle+|r e\rangle|10\rangle) .
$$

We omit in the following discussion overall phase factor introduced to the total state by reflections from mirrors $M$. After the second beam splitter 
$\left(B S_{2}\right)$, the total state reads

$$
|\phi\rangle_{\text {out }}=\frac{i}{\sqrt{2}}\left(\left|\psi^{+}\right\rangle|01\rangle+\left|\psi^{-}\right\rangle|10\rangle\right),
$$

where $\left|\psi^{ \pm}\right\rangle=\frac{1}{\sqrt{2}}(|r e\rangle \pm i|e r\rangle)$. Conditional on a single click at photodetectors $D_{+}$or $D_{-}$, the atomic ensembles are projected onto a maximally entangled state. After establishing entanglement, the qubits are transferred to their computational basis states $|0\rangle_{L} \equiv|g\rangle$ and $|1\rangle_{L} \equiv|s\rangle$ by classical optical pulses $\Omega_{g}$ and $\Omega_{s}$. Ideally every run of the protocol gives an entangled state of two atomic ensembles with success probability $p_{\text {success }}=\eta$, where $\eta=\eta_{D} \eta_{S}^{2}$ is the combined detection and source efficiency. This is a significant improvement compared to the success probability $p_{\text {success }}=\eta^{2} / 2$ of the double-heralding protocol in Ref. [51].

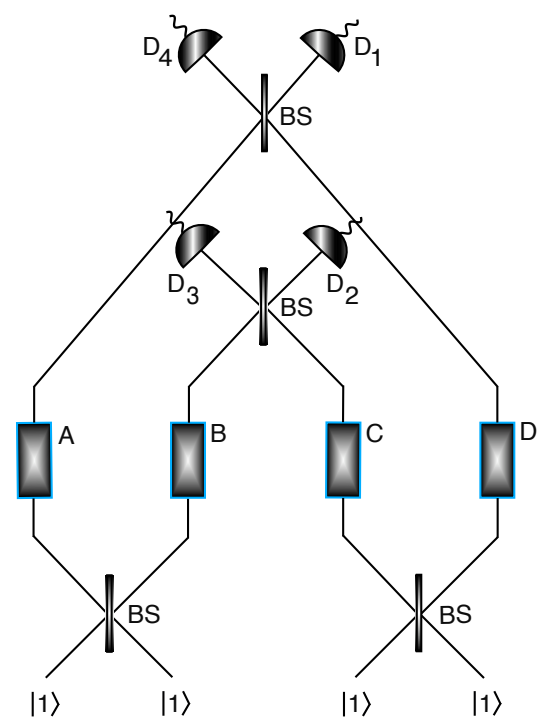

Figure 9: The scheme for creating the 4-qubit GHZ state. Four ensembles $A, B, C$, and $D$ prepared in the state $|\phi\rangle_{A B C D}=|e e e e\rangle$ interact with two pairs of entangled, indistinguishable photons. Conditional on photodetector clicks at the photodetector pair $\left(D_{1}, D_{2}\right),\left(D_{1}, D_{3}\right),\left(D_{4}, D_{2}\right)$ or $\left(D_{4}, D_{3}\right)$, the state of four qubits is projected onto the 4-qubit GHZ state (up to phase correcting operations) with success probability $p_{\text {success }}=\eta^{2} / 2$. 


\subsection{Generation of the GHZ and cluster states}

The entangling operation can be used to efficiently create arbitrary cluster states of any degree of connectivity, including a 2D universal resource states for one-way quantum computer. However, a modification of the entangling procedure yields an even more dramatic improvement in the efficiency of cluster state generation. By arranging the atomic ensembles in a four-mode interferometer as shown in Fig. 9, the detection of two photons will create the four-qubit GHZ state in a single step with the success probability $p_{\text {success }}=\eta^{2} / 2$. Moreover, since only two photons are detected, the protocol is relatively insensitive to detector losses. Higher GHZ states can be created by a straightforward extension. A subsequent GHZ states are generated with success probability

$$
p_{\text {success }}=\eta^{Q / 2}(Q-2) / 2^{Q-2},
$$

where $Q=4,6, \ldots$ is the number of the qubits.

As already mentioned, the GHZ states are locally equivalent to the cluster states. The efficiently generated large GHZ states may serve as building blocks for universal graph states. By entangling small clusters with the above entangling procedure, large cluster states can be constructed. A single photon applied to a pair of qubits (each from two different 4-qubit cluster states) followed by a single photodetector click creates an 8-qubit cluster state with success probability $p_{\text {success }}=\eta^{\prime} / 8$. This procedure can be repeated in an efficient manner.[69] In the case of failure, the two qubits that participated in linking are measured in the computational basis, and the rest of the cluster state is recycled.[70]

\section{Errors, decoherence mechanisms and fidelity}

The dominant errors and decoherence mechanisms that enter the entangling operation are the following:

- the coincident event in the HOM effect,

- the spontaneous emission rate of the Rydberg state,

- the black-body transfer rate (to other Rydberg states),

- the atomic collision rate,

- the doubly-excited Rydberg states and singly-excited states outside the desired two-level system, 
- no absorption event,

- the inefficiency and the dark count rate of the photodetectors.

We analyze in more detail the above dominant error and decoherence mechanisms on the following experimental implementation. First, let us consider the coincident events in the HOM effect. The single indistinguishable photons that recombine at the first beam splitter $\left(B S_{1}\right)$ can be generated by means of spontaneous parametric down-conversion (SPDC) process or sources of single-photon pulses such as atomic ensemble inside an optical cavity.[71, 72] The SPDC source (the non-linear crystal) must be pumped with a narrowband $(\sim 1 \mathrm{MHz})$ laser or placed inside a cavity. These kind of cavity-enhanced SPDC sources produce pairs of identical photons with a narrow bandwidth of order of $\mathrm{MHz}$ and a spectral brightness of $\sim 1500$ photons/s per MHZ bandwidth.[73, 74]

In general, successful generation of the entangled state of light depends on the proper setup, where both photons from the SPDC source recombine at $B S_{1}$ at the same time. In a recent experiment, the coincident event in the HOM effect occurs with a low rate of 1500 counts/s.[75] In fact, it is possible to completely eliminate the coincident event in the HOM effect by getting rid of the $B S_{1}$. In place of single-photon sources and $B S_{1}$, one can use a SPDC source generating pairs of single-photons entangled in momentum (path) degree of freedom.[76, 77] The state of the photons is given by $|\phi\rangle_{\text {light }}=\frac{1}{\sqrt{2}}\left(|1,1 ; 0,0\rangle_{A ; B}+|0,0 ; 1,1\rangle_{A ; B}\right)$, where states $|1,1 ; 0,0\rangle_{A ; B}$ and $|0,0 ; 1,1\rangle_{A ; B}$ represent two single photons propagating along slightly different paths through upper and lower arm of the interferometer, and interacting with atomic ensembles $A$ and $B$, respectively (see Fig. 10). State $|\phi\rangle_{\text {light }}$ represents so-called dual-rail qubit encoding. Moreover, since the SPDC process is a phase and energy matching phenomenon, no phase difference appears between two paths (pairs) $A$ and $B$.[76] In general, the whole Mach-Zehnder interferometer needs to be phase-stable. In the case of a GHZ state generation, phase locking of a large number of Mach-Zehnder interferometers is very demanding (although possible). Therefore, by replacing single-photon sources and $B S_{1}$ with the SPDC source generating entangled photon pairs we may simplify experimental realization of the entangling operation (although the second half of the interferometer after atomic ensembles still requires phase stabilization). Recently, it has been shown that these kind of entangled pairs of photons can be generated very effectively.[77]

Now, assume that an atomic vapor consists of $500{ }^{87} \mathrm{Rb}$ atoms placed in the far off-resonant optical trap (FORT) or magneto-optical trap (MOT). 


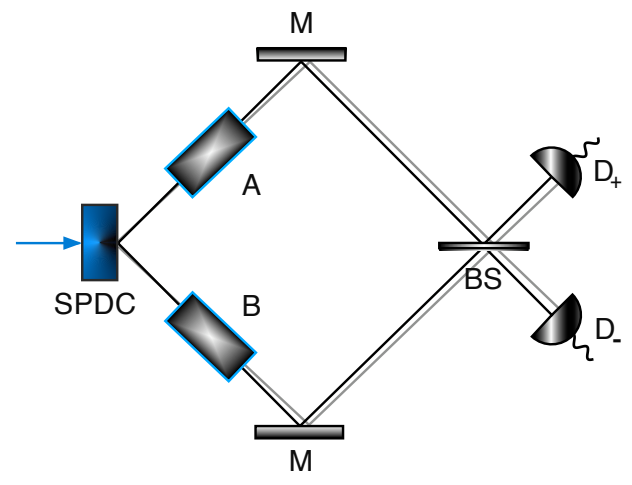

Figure 10: Example of an experimental implementation of the entangling protocol. The source of a single-photon pair entangled in the momentum (path) degree of freedom consists of the type I nonlinear crystal.

The atomic levels $|g\rangle,|e\rangle$, and $|r\rangle$ may correspond to $\left(5 S_{1 / 2}, F=1\right),\left(5 P_{3 / 2}, F=\right.$ $2)$ and $43 D_{5 / 2}$ or $58 D_{3 / 2}$, respectively. State $|s\rangle$ may correspond to the hyperfine state $\left(5 S_{1 / 2}, F=2\right)$, which implies that the transition from $|s\rangle$ to $|r\rangle$ is a two-photon process (see Fig. 5). We have identified state $|e\rangle$ with a short lived state $\left(5 P_{3 / 2}, F=2\right)$, when in fact it must be a long lived energy level. However, in case of the MOT, the requirement of a long relaxation time of the state $|e\rangle$ can be lifted since the trap lasers produce a constant population in the $|e\rangle$ state. [35, 78] In general, a requirement of state $|e\rangle$ is imposed to simplify experimental realization of the protocol where usually two-photon excitation are used to obtain Rydberg atoms. The spatial distribution of an atomic cloud is a quasi one-dimensional (cigar shaped) ensemble with probability density given by Eq. (29). Atomic vapors described with quasi onedimensional probability density have been demonstrated experimentally.[37] When a protocol is based on a quantum optical system, its performance is limited by the inefficiency and the dark count rate of the photodetectors. The dark count rate of a modern photodetector $\gamma_{d c}$ can be as low as 20 $\mathrm{Hz}$ and efficiency reaches $\eta_{D} \approx 30 \%$ for wavelengths around $480 \mathrm{~nm}$. The probability of the dark count is $P_{d c}=1-\exp \left(-\gamma_{d c} t / p_{\text {success }}\right)$, where $t$ is the time scale of the entangling protocol. In general, the probability of the dark count is negligible for $p_{\text {success }}>\gamma_{d c} t$.

Since the length of the atomic ensemble needs to be of an order of several $\mu \mathrm{m}$, the most important source of errors is the lack of absorption event. The probability of an absorption of a single photon by a cigar shaped atomic en- 
semble is given by $P_{a b s} \cong 1-e^{-N_{i} \sigma_{0} / A}$, with $N_{i}=N$ the number of atoms in the interaction region, $\sigma_{0}=3 \lambda^{2} \gamma_{0} /(2 \pi \gamma)$ is the on-resonance scattering cross section of a single-photon pulse, where $\gamma_{0}$ is the spontaneous decay rate of the Rydberg state to low-lying levels and $\gamma$ is the spontaneous decay to other Rydberg states. $[2,66,79] A=\pi w_{0}^{2}$ is the area of a single-photon pulse with a waist $w_{0} \approx \pi \lambda$.[80] With $\lambda_{43 D}=485.766 \mathrm{~nm}, \gamma_{0}=1.110^{4}$ $\mathrm{Hz}$ and $\gamma=7.210^{4} \mathrm{~Hz}$, the probability of an absorption for $43 D_{5 / 2}$ state is $P_{a b s} \cong 0.69$. For $\lambda_{58 D}=485.081 \mathrm{~nm}, \gamma_{0}=4.810^{3} \mathrm{~Hz}$ and $\gamma=2.010^{4} \mathrm{~Hz}$, the probability of an absorption for $58 D_{3 / 2}$ state is $P_{a b s} \cong 0.84$. The probability of an absorption for both Rydberg states is much too low for reliable operation of the entangling gate, therefore one has to use atomic ensembles with larger number of atoms $N$. A smaller area $A$ does not improve the probability of an absorption since it implies smaller number of atoms $N_{i}$ in the interaction region. In fact, the optimal area $A$ coincides with a size of an atomic ensemble in transverse directions. Consequently, for a level structure shown in Fig. 5 the only solution to a low probability of an absorption is higher number of atoms $N$. To render probability of absorption close to unity, one has to use more than 2500 atoms. High fidelity dipole blockade in such a large ensemble is not feasible.[79] Another potential difficulty may be a rather high atomic density of the sample. Several $\mu \mathrm{m}$ long atomic vapors consisting of thousand of atoms are hard to prepare.

To overcome these difficulties, we propose different experimental implementation based on a level structure shown in Fig. 11. This level structure implements the entangling operation but in slightly different manner. Here, the dipole blockade is not used to block absorption of a second photon. In this implementation, we employ the dipole blockade mechanism to prepare a single atomic excitation in the Rydberg state $\left|r_{1}\right\rangle$ by means of a two-photon process. This is precisely a bit flip operation $X$ described in Sec. 7. However here, the two-photon Rabi frequency of a laser field that realizes a $\pi$ pulse between states $|g\rangle$ and $\left|r_{1}\right\rangle$ is given by $\Omega=\Omega_{g e} \Omega_{e r_{1}} / 2 \Delta$ where $\Delta$ is a small detuning (not shown in Fig. 11). For $\left|r_{1}\right\rangle=43 D_{5 / 2}$ a bit flip operation can be carried out on a microsecond timescale. Subsequently, two single photons interact with the atomic ensembles: One and only one photon is absorbed by the Rydberg atom in the state $\left|r_{1}\right\rangle$. The Rydberg atom is excited to the state $\left|r_{2}\right\rangle$. We assume that the probability of a two-photon absorption process is negligible since both photons are on-resonance with a transition between Rydberg states $\left|r_{1}\right\rangle$ and $\left|r_{2}\right\rangle$.[81, 82, 83] Finally, following a single photodetector click, two $\pi$ pulses realized by a classical fields are applied simultaneously to the transitions between the second Rydberg level $\left|r_{2}\right\rangle$ and the state $|s\rangle$ (it may possibly be a two-photon process), and between the first 


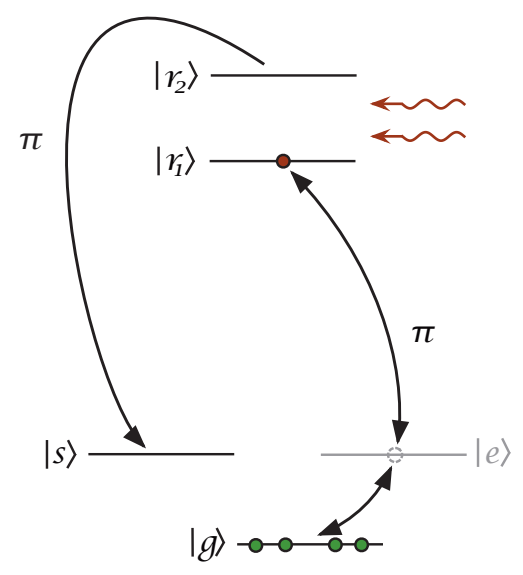

Figure 11: Relevant atomic level structure with allowed atomic transitions. The low-lying state $|g\rangle$ is coupled to the first Rydberg state $\left|r_{1}\right\rangle$ through intermediate low-lying level $|e\rangle$ by means of a classical field that implements a $\pi$ pulse. A second $\pi$ pulse realized by a classical field is applied to the transition between the second Rydberg level $\left|r_{2}\right\rangle$ and the state $|s\rangle$ (it may possibly be a two-photon process).

Rydberg level $\left|r_{1}\right\rangle$ and the state $|g\rangle$. The main strength of this implementation lies in a fact that all $\pi$ pulses that transfer single excitation between Rydberg states and low-lying storage states $|g\rangle$ and $|s\rangle$ are highly reliable operations with fidelity $F_{\text {single }} \cong 0.99$. Most importantly, this kind of control over atomic ensembles have been demonstrated experimentally. [37, 84, 85] Consequently, this implementation of the entangling operation requires relatively straightforward experimental extension of known procedures.

Let us now examine if the probability of absorption of a single photon by a single Rydberg atom is high enough for reliable operation of our entangling gate. The two single photons couple to the transition between level $|r\rangle=45 P_{3 / 2}$ (not shown in Fig. 11) and level $\left|r_{2}\right\rangle=58 D_{3 / 2}$. The reader should note that state $\left|r_{1}\right\rangle=43 D_{5 / 2}$ is only used in a bit flip operation $X$ and then single Rydberg atom is excited by means of a fast microwave pulse to the Rydberg state $|r\rangle=45 P_{3 / 2}$. For $\lambda_{45 P-58 D}=370.783 \mu \mathrm{m}$, $\gamma_{0}=4.810^{3} \mathrm{~Hz}$ and $\gamma=2.010^{4} \mathrm{~Hz}$, the probability of an absorption $P_{a b s} \cong 1-e^{-2 \sigma_{0} / A} \cong 0.90$, where area $A=0.1 \lambda_{45 P-58 D}^{2}$ (this implies a waist of a single-photon pulse $w_{0} \cong 66 \mu \mathrm{m}$ ). [86] High probability of an absorption requires strongly focused light fields with small area $A$.[87, 88, 89] The improved ratio of $\sigma_{0} / A$ and therefore higher probability of an absorption for 
this experimental implementation is owned to the stronger focusing relative to the wavelength of a single-photon pulse. Naturally, the focusing regime is limited by a size of the atomic sample and diffraction limited area of a single-photon pulse. [87, 88, 89] To render the probability of an absorption close to unity one may apply a mode converter (shaper) to a single-photon fields. [90] The probability of an absorption depends also on the spontaneous decay rates associated with the Rydberg state. The above level assignment is arbitrary. Rich structure of Rydberg levels offers many possible ways for level assignment, therefore one may be able to choose two Rydberg states with higher on-resonance scattering cross section. The overall time scale of the entangling protocol $t$ consists of a time required by the $\pi$ pulse $t_{\pi}=11.2$ $\mu \mathrm{s}$ (preparation of a single Rydberg atom in the state $\left|r_{1}\right\rangle$ ) and time of an interaction part of the protocol given by

$$
t_{45 P-58 D}=\frac{\pi}{2(\sqrt{2} g)} \cong 2.9 \mathrm{~ns}, \text { with } g=\sqrt{\frac{\sigma_{0} \gamma c}{4 V}},
$$

where $g$ is the atom-light coupling constant and $V=A L$ is the interaction volume with $L=12 \mu \mathrm{m}$ being the length of an atomic medium.[2] After successful entanglement preparation the state of atomic ensembles is quickly stored in the long lived atomic states $|g\rangle$ and $|s\rangle$ in time significantly shorter than time $t_{\pi}$. In summary, the entangling protocol can be carried out on a microsecond time scale.

The two single photons employed in the entangling procedure belong to the far-infrared part of the electromagnetic spectrum. The photodetectors sensitive to this part of the spectrum are under development. The detection range of quantum dot infrared photodetectors such as $\mathrm{In}(\mathrm{Ga})$ As quantum ring terahertz photodetector reaches $175 \mu \mathrm{m}$. Another photodetector operating in $\mathrm{THz}$ regime is based on hot-electron effect in nanobolometers and used in astrophysics for registration of the Universe radiation.[91] A bolometer is a device that measures the energy of incident electromagnetic radiation. Although photodetector based on nanobolometers is characterized with rather complex fabrication and has to work in ultra-cold temperature regime (around $200 \mathrm{mK}$ ), it is highly sensitive and capable of detecting single $\mathrm{THz}$ photons with quantum efficiency close to $100 \%$ (maintaining at the same time low dark count rate).[91]

The source of single-photon pulses in far-infrared frequency regime can be based on atomic ensemble or single ion placed inside an optical cavity. Preferably, the single-photon sources should work on-demand. However as already mentioned, one may choose different Rydberg levels and implement 
entangling gate with single photons from less extreme part of the electromagnetic spectrum.

The spontaneous emission from the Rydberg state and the black-body transfer (to other Rydberg states) occur with rates of order $10^{3} \mathrm{~Hz}$ (or even $10^{2} \mathrm{~Hz}$ for higher Rydberg states), and are negligible, since following successful entanglement preparation the state of matter qubit is quickly stored in the long lived atomic states $|g\rangle$ and $|s\rangle$. Exact values of these rates are given in Ref. [66]. The atomic collision rate inside atomic vapor is given by

$$
\tau_{c o l}^{-1} \approx n \sigma_{c o l} / \sqrt{M / 3 k_{B} T}
$$

with $n$ the number density of atoms, $\sigma_{c o l}$ the collisional cross section $(\sim$ $\left.10^{-14} \mathrm{~cm}^{2}\right), M$ the atomic mass, $k_{B}$ the Boltzmann's constant, and $T$ the temperature.[32] Assuming a vapor with the number density of atoms of order $10^{12} \mathrm{~cm}^{-3}$ and the temperature of $\sim 10^{-3} \mathrm{~K}$, the atomic collision rate can be as low as $2 \mathrm{~Hz}$. Moreover, with a sufficiently large energy difference between states $|g\rangle$ and $|s\rangle$ a single collision is not likely to affect the qubit.

A low temperature of an atomic vapor implies negligible Doppler broadening. The Doppler broadening is described by the Gaussian distribution with a standard deviation of $\Delta \lambda=\lambda_{0} \sqrt{k_{B} T / M c^{2}}$ where $\lambda_{0}$ is the center wavelength of the Doppler profile (wavelength of a transition between states $|r\rangle$ and $\left.\left|r_{1}\right\rangle\right)$. For $\lambda_{0}=\lambda_{45 P-58 D}$, the Doppler broadening is $\Delta \lambda=0.410^{-6}$ $\mu \mathrm{m}$. Therefore, the Doppler broadening does not affect fidelity of the entangling protocol.

Considering both the overall and interaction time scale of the protocol, the entangling procedure is mostly affected by the no absorption event assuming high quantum efficiency and low dark count rate of $\mathrm{THz}$ photodetectors. We assume that the coincident event rate in the HOM effect and two-photon absorption process are negligible. In the presence of the above noise and decoherence mechanisms, the final state of the system conditional on a single photodetector click is given by

$$
\rho_{\text {fin }}=(1-2 \varepsilon)\left|\psi^{ \pm}\right\rangle\left\langle\psi^{ \pm}\right|+2 \varepsilon \rho_{\text {noise }}+\mathcal{O}\left(\varepsilon^{2}\right),
$$

where $\left|\psi^{ \pm}\right\rangle=\frac{1}{\sqrt{2}}(|s g\rangle \pm i|g s\rangle)$ and $\varepsilon=1-P_{a b s}$ where $P_{a b s}$ is the probability of an absorption of a single photon by a single Rydberg atom. $\rho_{\text {noise }}$ denotes the unwanted terms in the state of the two atomic ensembles. It is worth noting that the source efficiency does not affect the fidelity of the final state, it only lowers the success probability. After taking into account all dominant error mechanisms, the fidelity of the prepared entangled state is given by

$$
F=\left\langle\psi^{ \pm}\left|\rho_{\text {fin }}\right| \psi^{ \pm}\right\rangle \cong 0.90 .
$$


Stronger focusing regime and/or application of a mode converter (shaper) to a single-photon fields should render fidelity of the entangling operation close to current fault-tolerant thresholds of the topological codes.[92, 93, 94, 95] As already stated, a new entangling protocol is capable of creating cluster states of any degree of connectivity. Since a 3D cluster lattice can be used to efficiently implement planar surface codes, one can exploit the topological error-correction capabilities of these codes to perform fault-tolerant quantum computation with our entangling procedure. One can also increase fidelity of the final states with a use of purification techniques for a price of cluster size reduction when the purification fails. [96, 97]

\subsection{Polarization-entangled photon pair}

The dual-rail qubit encoding responsible for significant experimental simplification of the protocol is equivalent to the polarization-entangled state of a photon pair $|\phi\rangle_{\text {light }}=\frac{1}{\sqrt{2}}\left(|H V\rangle_{A B}+|V H\rangle_{A B}\right)$, where $H$ and $V$ are the horizontal and vertical polarizations of a photon, respectively (see Fig. 12). A polarization-entangled photon pairs are generated by means of the type I nonlinear crystal.[76, 98] A pair of photons entangled in a polarization degree of freedom allow us to simplify the experimental implementation of the protocol even further. In fact, the dipole blockade mechanism is no longer required for a reliable operation of our entangling protocol. Here, the entangling gate works as follows. Initially, we prepare each ensemble $A$ and $B$ in the collective ground state $|g\rangle \equiv\left|g_{1}, g_{2}, \ldots, g_{N}\right\rangle$, where the ground state $|g\rangle$ may correspond to atomic level $\left(5 S_{1 / 2}, F=1\right)$. Subsequently, one of the polarization-entangled photons, for instance horizontally polarized, interacts with the atomic ensembles: One and only one atom in the ensemble is excited by the photon to the excited state $|e\rangle$ that may correspond to atomic level $\left(5 P_{3 / 2}, F=2\right)$. The absorption of the second photon (the vertically polarized) is completely prohibited since the polarization beam splitter placed in front of each ensemble prevents vertically polarized photon from interacting with atomic vapors. Following a balanced beam splitter $(B S)$ interaction and conditionally on a photodetector click, the state of atomic ensembles is projected onto a pure maximally entangled state. The main advantage of this implementation lies in a fact that the second photon is never absorbed by atomic ensembles and the probability of an absorption of the first photon $P_{a b s} \cong 1-e^{-N_{i} \sigma_{0} / A}$ can practically reach unity since one can exploit optically thick atomic vapors, i.e., highly dense and/or large vapors. Therefore, a single photon should easily couple to atomic medium. Moreover, we are no longer limited by a size of the atomic ensemble which 


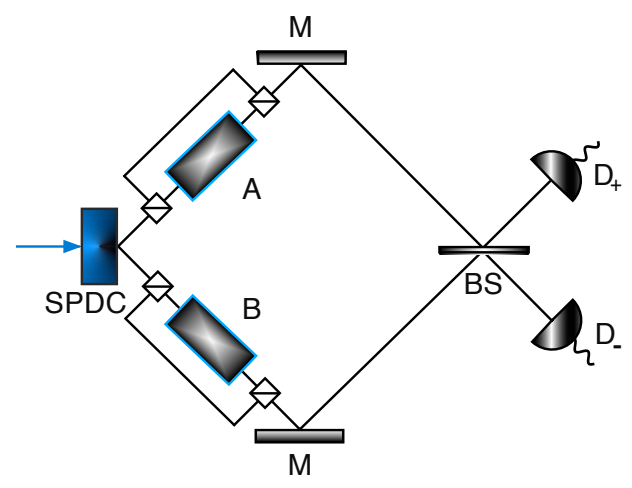

Figure 12: Example of an experimental implementation of the entangling protocol exploiting the polarization-entangled photon pair. The source of a single-photon pair consists of the type I nonlinear crystal. A "square" placed in front of and behind each ensemble depicts the polarization beam splitter.

significantly simplifies the preparation of atomic samples.

The dominant errors and decoherence mechanisms that enter the entangling operation based on experimental implementation exploiting the polarization-entangled photon pair are the following:

- the spontaneous emission rate of the exited state $|e\rangle$,

- the atomic collision rate,

- the inefficiency and the dark count rate of the photodetectors.

The atomic level $\left(5 P_{3 / 2}, F=2\right)$ corresponding to the excited state $|e\rangle$ is a short-lived level. Therefore, one has to apply additional laser pulse between the excited state $|e\rangle$ and storage state $|s\rangle$, exploiting in fact properties of the stimulated Raman adiabatic passage, to reliably carry out entangling operation. The atomic collision rate as described in the previous section is insignificant. The dark count rate of a modern photodetector $\gamma_{d c}$ can be negligibly low $(20 \mathrm{~Hz})$ with efficiency reaching $\eta_{D} \approx 90 \%$ for wavelengths around 780 $\mathrm{nm}$. Considering all the dominant errors and decoherence mechanisms, an implementation of the entangling protocol exploiting polarization-entangled photon pair can generate entangled states of atomic ensembles with fidelity $F \cong 1$ and success probability $p_{\text {success }}=\eta$, where $\eta=\eta_{D} \eta_{S}^{2}$ is the combined detection and source efficiency. It is worth noting that the fidelity of the 
final state does not depend on $\eta$. More importantly, all results associated with generation of the GHZ and cluster states follow exactly.

New entangling operation presented here allow us to map an entangled state of a photon pair on two macroscopic atomic ensembles in a heralded fashion. In general, one may imagine an experiment in which a multi-qubit photonic cluster state is mapped on a collection of atomic ensembles (see Fig. 13). In this way, a possibly large $Q$-qubit cluster state can be reliably

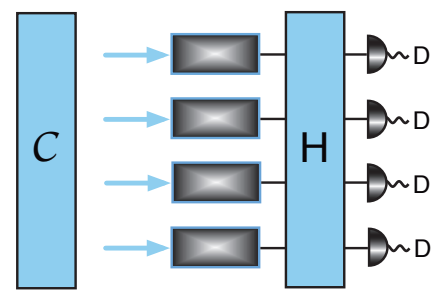

Figure 13: A mapping of cluster state on a collection of atomic ensembles. $H$ depicts a multi-port beam splitter that erases which path information.

stored in $Q$ atomic vapors.

\section{Conclusions}

We have reviewed in some detail electromagnetically induced transparency technique, Raman interaction and associated propagation phenomena. EIT technique allows us to induce coherent behavior of a macroscopic atomic medium under certain conditions. The reduced group velocity and dark state-polaritons are a remarkable propagation phenomena associated with propagation of an optical pulse in atomic medium under EIT conditions that has lead to the concept of quantum memory. In principle, an atomic medium is capable of storing single-photon pulses. Apart from EIT, one can employ Rydberg atoms and dipole blockade mechanism to induce collective behavior of atomic vapor. On the basis of the above techniques, probabilistic entanglement generation between atomic vapors is feasible. We have reviewed several schemes for probabilistic entanglement generation such as DLCZ protocol and double-heralding protocol. We have also presented and studied a new scheme for cluster state generation based on atomic ensembles and the dipole blockade mechanism. A new entangling protocol consists of single-photon sources, ultra-cold atomic ensembles, and regular photodetectors. The protocol generates in a single step GHZ state 
with success probability $p_{\text {success }} \sim \eta^{Q / 2}$, where $Q$ is the number of the qubits, and high fidelity $F \cong 0.90$ (or $F \cong 1$ when polarization-entangled implementation is used). Our new entangling gate is more efficient than any previously proposed probabilistic scheme with realistic photodetectors and single-photon sources. Every run of the procedure gives an entangled state of two atomic ensembles with success probability $p_{\text {success }}=\eta$, where $\eta$ is the combined detection and source efficiency. The double-heralding protocol produces an entangled state of two matter qubits with the success probability $p_{\text {success }}=\eta^{2} / 2$. The protocol proposed by Lim et al. [63] requires on average two repetitions to realize the desired gate operation. Moreover, the successful implementation of this protocol requires detection of a photon pair. A new entangling protocol requires only single photon detection. In general, number-resolution photodetectors are not required. However, a reliable photon counting detector with low dark count rate would be able to herald any error in the procedure increasing the fidelity close to unity. The GHZ states are locally (up to Hadamard operation) equivalent to starshaped cluster states. The efficiently generated large GHZ states may serve as building blocks for universal graph states.

We have also reviewed and analyzed a scheme implementing any single-qubit operation on the qubit defined as collective states of mesoscopic ensemble.

The scheme for single-qubit rotations is based on classical optical pulses and the dipole blockade mechanism. The experimental implementation may be carried out with high fidelity $F_{\text {single }} \cong 0.99$ and on the microsecond timescale with current state-of-the-art experimental setups.

The described protocols for single-qubit rotations and entangling operation open a possibility of experimental implementation of the measurement-based quantum computer based on atomic ensembles.

\section{Acknowledgments}

We thank Charles Adams, Matthew Jones, Klaus Mølmer and Mark Saffman for helpful discussions. This work was supported by the White Rose Foundation, and the National Research Foundation and Ministry of Education, Singapore.

\section{References}

[1] L.-M. Duan, M. D. Lukin, J. I. Cirac and P. Zoller. Long-distance quantum communication with atomic ensembles and linear optics. Nature, 
414:413, 2001.

[2] K. Hammerer, A. S. Sørensen and E. S. Polzik. Quantum interface between light and atomic ensembles. e-print arXiv:quant-ph/080\%.3358v3, 2009.

[3] M. Fleischhauer and A. Imamoğlu and J. P. Marangos. Electromagnetically induced transparency: Optics in coherent media. Rev. Mod. Phys., 77:633, 2005.

[4] M. D. Lukin. Colloquium: Trapping and manipulating photon states in atomic ensembles. Rev. Mod. Phys., 75:457, 2003.

[5] S. D. Barrett, P. P. Rohde and T. M. Stace. Scalable quantum computing with atomic ensembles. e-print arXiv:quant-ph/0804.0962v1, 2008.

[6] S. Manz, T. Fernholz, J. Schmiedmayer and J.-W. Pan. Collisional decoherence during writing and reading quantum states. Phys. Rev. A, 75:040101(R), 2007.

[7] I. Novikova, A. V. Gorshkov, D. F. Phillips, A. S. Sørensen, M. D. Lukin and R. L. Walsworth. Optimal control of light pulse storage and retrieval. Phys. Rev. Lett., 98:243602, 2007.

[8] R. Pugatch, M. Shuker, O. Firstenberg, A. Ron and N. Davidson. Topological stability of stored optical vortices. Phys. Rev. Lett., 98:203601, 2007.

[9] T. Hong, A. V. Gorshkov, D. Patterson, A. S. Zibrov, J. M. Doyle, M. D. Lukin and M. G. Prentiss. Realization of coherent optically dense media via buffer-gas cooling. Phys. Rev. A, 79:013806, 2009.

[10] S. E. Harris, J. E. Field and A. Imamoğlu. Nonlinear optical processes using electromagnetically induced transparency. Phys. Rev. Lett., 64:1107, 1990.

[11] S. E. Harris. Electromagnetically induced transparency. Phys. Today, 50:36, 1997.

[12] R. G. Beausoleil, W. J. Munro and T. P. Spiller. Applications of coherent population transfer to quantum information processing. Topic Review in JMO, 51:1559, 2004.

[13] E. Arimondo. Coherent population trapping in laser spectroscopy. Progress in Optics, 35:259, 1997. 
[14] K.-J. Boller, A. Imamoğlu and S. E. Harris. Observation of electromagnetically induced transparency. Phys. Rev. Lett., 66:2593, 1991.

[15] M. Fleischhauer and M. D. Lukin. Dark-state polaritons in electromagnetically induced transparency. Phys. Rev. Lett., 84:5094, 2000.

[16] S. E. Harris, J. E. Field and A. Kasapi. Dispersive properties of electromagnetically induced transparency. Phys. Rev. A, 46:R29, 1992.

[17] P. Kok and B. W. Lovett. Introduction to Optical Quantum Information Processing. Cambridge University Press, in press.

[18] C. Liu, Z. Dutton, C. H. Behroozi and L. V. Hau. Observation of coherent optical information storage in an atomic medium using halted light pulses. Nature, 409:490, 2001.

[19] D. F. Phillips, A. Fleischhauer, A. Mair, R. L. Walsworth and M. D. Lukin. Storage of light in atomic vapor. Phys. Rev. Lett., 86:783, 2001.

[20] A. V. Turukhin, V. S. Sudarshanam, M. S. Shahriar, J. A. Musser, B. S. Ham and P. R. Hemmer. Observation of ultraslow and stored light pulses in a solid. Phys. Rev. Lett., 88:023602, 2001.

[21] L. V. Hau, S. E. Harris, Z. Dutton and C. H. Behroozi. Light speed reduction to 17 metres per second in an ultracold atomic gas. Nature, 397:594, 1999.

[22] M. D. Lukin, S. F. Yelin and M. Fleischhauer. Entanglement of atomic ensembles by trapping correlated photon states. Phys. Rev. Lett., $84: 4232,2000$.

[23] M. Fleischhauer and M. D. Lukin. Quantum memory for photons: Dark-state polaritons. Phys. Rev. A, 65:022314, 2002.

[24] C. Mewes and M. Fleischhauer. Two-photon linewidth of light "stopping" via electromagnetically induced transparency. Phys. Rev. A, 66:033820, 2002.

[25] A. Kuzmich, W. P. Bowen, A. D. Boozer, A. Boca, C. W. Chou, L.M. Duan and H. J. Kimble. Generation of nonclassical photon pairs for scalable quantum communication with atomic ensembles. Nature, 423:731, 2003. 
[26] C. H. van der Wal, M. D. Eisaman, A. André, R. L. Walsworth, D. F. Phillips, A. S. Zibrov and M. D. Lukin. Atomic memory for correlated photon states. Science, 301:196, 2003.

[27] J. Laurat, K. S. Choi, H. Deng, C. W. Chou and H. J. Kimble. Heralded entanglement between atomic ensembles: Preparation, decoherence, and scaling. Phys. Rev. Lett., 99:180504, 2007.

[28] D. Felinto, C. W. Chou, H. de Riedmatten, S. V. Polyakov and H. J. Kimble. Control of decoherence in the generation of photon pairs from atomic ensembles. Phys. Rev. A, 72:053809, 2005.

[29] T. Chanelière, D. N. Matsukevich, S. D. Jenkins, S.-Y. Lan, T. A. B. Kennedy and A. Kuzmich. Storage and retrieval of single photons transmitted between remote quantum memories. Nature, 438:833, 2005.

[30] M. D. Eisaman, A. André, F. Massou, M. Fleischhauer, A. S. Zibrov and M. D. Lukin. Electromagnetically induced transparency with tunable single-photon pulses. Nature, 438:837, 2005.

[31] K. S. Choi, H. Deng, J. Laurat and H. J. Kimble. Mapping photonic entanglement into and out of a quantum memory. Nature, 452:67, 2008.

[32] D. F. V. James and P. G. Kwiat. Atomic-vapor-based high efficiency optical detectors with photon number resolution. Phys. Rev. Lett., 89:183601, 2002.

[33] A. Imamoğlu. High efficiency photon counting using stored light. Phys. Rev. Lett., 89:163602, 2002.

[34] T. F. Gallagher. Rydberg Atoms. Cambridge University Press, 1994.

[35] W. Li, I. Mourachko, M. W. Noel and T. F. Gallagher. Millimeterwave spectroscopy of cold Rb Rydberg atoms in a magneto-optical trap: Quantum defects of the ns, np, and nd series. Phys. Rev. A, 67:052502, 2003.

[36] C. Gerry and P. Knight. Introductory Quantum Optics. Cambridge University Press, 2005.

[37] T. A. Johnson, E. Urban, T. Henage, L. Isenhower, D. D. Yavuz, T. G. Walker and M. Saffman. Rabi oscillations between ground and Rydberg states with dipole-dipole atomic interactions. Phys. Rev. Lett., 100:113003, 2008. 
[38] C. S. E. van Ditzhuijzen, A. F. Koenderink, J. V. Hernández, F. Robicheaux, L. D. Noordam and H. B. van Linden van den Heuvell. Spatially resolved observation of dipole-dipole interaction between Rydberg atoms. Phys. Rev. Lett., 100:243201, 2008.

[39] T. G. Walker and M. Saffman. Zeros of Rydberg-Rydberg Föster interactions. J. Phys. B, 38:S309, 2005.

[40] R. C. Stoneman, M. D. Adams and T. F. Gallagher. Resonant-collision spectroscopy of Rydberg atoms. Phys. Rev. Lett., 58:1324, 1987.

[41] T. G. Walker and M. Saffman. Consequences of Zeeman degeneracy for the van der Waals blockade between Rydberg atoms. Phys. Rev. A, 77:032723, 2008.

[42] D. Jaksch, J. I. Cirac, P. Zoller, S. L. Rolston, R. Côté and M. D. Lukin. Fast quantum gates for neutral atoms. Phys. Rev. Lett., 85:2208, 2000.

[43] M. D. Lukin, M. Fleischhauer, R. Côté, L.-M. Duan, D. Jaksch, J. I. Cirac and P. Zoller. Dipole blockade and quantum information processing in mesoscopic atomic ensembles. Phys. Rev. Lett., 87:037901, 2001.

[44] E. Brion, K. Mølmer and M. Saffman. Quantum computing with collective ensembles of multilevel systems. Phys. Rev. Lett., 99:260501, 2007.

[45] I. Friedler, D. Petrosyan, M. Fleischhauer and G. Kurizki. Long-range interactions and entanglement of slow single-photon pulses. Phys. Rev. A, 72:043803, 2005.

[46] A. K. Mohapatra, T. R. Jackson and C. S. Adams. Coherent optical detection of highly excited Rydberg states using electromagnetically induced transparency. Phys. Rev. Lett., 98:113003, 2007.

[47] D. Petrosyan and M. Fleischhauer. Quantum information processing with single photons and atomic ensembles in microwave coplanar waveguide resonators. Phys. Rev. Lett., 100:170501, 2008.

[48] M. Zwierz and P. Kok. High-efficiency cluster-state generation with atomic ensembles via the dipole-blockade mechanism. Phys. Rev. A, 79:022304, 2009. 
[49] R. Raussendorf, D. E. Browne and H. J. Briegel. Measurement-based quantum computation on cluster states. Phys. Rev. A, 68:022312, 2003.

[50] M. Hein, J. Eisert and H. J. Briegel. Multiparty entanglement in graph states. Phys. Rev. A, 69:062311, 2004.

[51] S. D. Barrett and P. Kok. Efficient high-fidelity quantum computation using matter qubits and linear optics. Phys. Rev. A, 71:060310(R), 2005.

[52] S. J. van Enk, N. Lütkenhaus and H. J. Kimble. Experimental procedures for entanglement verification. Phys. Rev. A, 75:052318, 2007.

[53] C. W. Chou, S. V. Polyakov, A. Kuzmich and H. J. Kimble. Singlephoton generation from stored excitation in an atomic ensemble. Phys. Rev. Lett., 92:213601, 2004.

[54] C. W. Chou, H. de Riedmatten, D. Felinto, S. V. Polyakov, S. J. van Enk and H. J. Kimble. Measurement-induced entanglement for excitation stored in remote atomic ensembles. Nature, 438:828, 2005.

[55] C. W. Chou, J. Laurat, H. Deng, K. S. Choi, H. de Riedmatten, D. Felinto and H. J. Kimble. Functional quantum nodes for entanglement distribution over scalable quantum networks. Science, 316:1316, 2007.

[56] Z.-S. Yuan, Y.-A. Chen, B. Zhao, S. Chen, J. Schmiedmayer and J.-W. Pan. Experimental demonstration of a BDCZ quantum repeater node. Nature, 454:1098, 2008.

[57] Y.-A. Chen, S. Chen, Z.-S. Yuan, B. Zhao, C.-S. Chuu, J. Schmiedmayer and J.-W. Pan. Memory-built-in quantum teleportation with photonic and atomic qubits. Nature Physics, 4:103, 2008.

[58] P. Kok. Lecture notes on optical quantum computing. e-print arXiv:quant-ph/0705.4193v1, 2007.

[59] S. C. Benjamin. Comment on "Efficient high-fidelity quantum computation using matter qubits and linear optics". Phys. Rev. A, 72:056302, 2005.

[60] S. C. Benjamin, D. E. Browne, J. Fitzsimons and J. J. L. Morton. Brokered graph-state quantum computation. New J. Phys., 8:141, 2006. 
[61] D. L. Moehring, P. Maunz, S. Olmschenk, K. C. Younge, D. N. Matsukevich, L.-M. Duan and C. Monroe. Entanglement of single-atom quantum bits at a distance. Nature, 449:68, 2007.

[62] D. N. Matsukevich, P. Maunz, D. L. Moehring, S. Olmschenk and C. Monroe. Bell inequality violation with two remote atomic qubits. Phys. Rev. Lett., 100:150404, 2008.

[63] Y. L. Lim, A. Beige and L. C. Kwek. Repeat-until-success linear optics distributed quantum computing. Phys. Rev. Lett., 95:030505, 2005.

[64] Y. L. Lim, S. D. Barrett, A. Beige, P. Kok and L. C. Kwek. Repeatuntil-success quantum computing using stationary and flying qubits. Phys. Rev. A, 73:012304, 2006.

[65] M. Saffman and T. G. Walker. Creating single-atom and single-photon sources from entangled atomic ensembles. Phys. Rev. A, 66:065403, 2002 .

[66] J. O. Day, E. Brekke and T. G. Walker. Dynamics of low-density ultracold Rydberg gases. Phys. Rev. A, 77:052712, 2008.

[67] C. K. Hong, Z. Y. Ou and L. Mendel. Measurement of subpicosecond time intervals between two photons by interference. Phys. Rev. Lett., 59:2044, 1987.

[68] R. Ghosh and L. Mendel. Observation of nonclassical effects in the interference of two photons. Phys. Rev. Lett., 59:1903, 1987.

[69] K. Kieling, D. Gross and J. Eisert. Cluster state preparation using gates operating at arbitrary success probabilities. New J. Phys., 9:200, 2007.

[70] M. A. Nielsen. Optical quantum computation using cluster states. Phys. Rev. Lett., 93:040503, 2004.

[71] M. Halder, A. Beveratos, R. T. Thew, C. Jorel, H. Zbinden and N. Gisin. High coherence photon pair source for quantum communication. New J. Phys., 10:023027, 2008.

[72] J. K. Thompson, J. Simon, H. Loh and V. Vuletic. A high-brightness source of narrowband, identical-photon pairs. Science, 313:74, 2006. 
[73] J. S. Neergaard-Nielsen, B. M. Nielsen, H. Takahashi, A. I. Vistnes and E. S. Polzik. High purity bright single photon source. Opt. Express, 15:7940, 2007.

[74] X.-H. Bao, Y. Qian, J. Yang, H. Zhang, Z.-B. Chen, T. Yang and J.-W. Pan. Generation of narrow-band polarization-entangled photon pairs for atomic quantum memories. Phys. Rev. Lett., 101:190501, 2008.

[75] H. Kim, O. Kwon, W. Kim and T. Kim. Spatial two-photon interference in a Hong-Ou-Mandel interferometer. Phys. Rev. A, 73:023820, 2006.

[76] M. Barbieri, C. Cinelli, P. Mataloni and F. De Martini. Polarizationmomentum hyperentangled states: Realization and characterization. Phys. Rev. A, 72:052110, 2005.

[77] A. Rossi, G. Vallone, A. Chiuri, F. De Martini and P. Mataloni. Multipath entanglement of two photons. Phys. Rev. Lett., 102:153902, 2009.

[78] C. Monroe, W. Swann, H. Robinson and C. Wieman. Very cold trapped atoms in a vapor cell. Phys. Rev. Lett., 65:1571, 1990.

[79] M. Saffman. Private communication. 2009.

[80] M. Saffman and T. G. Walker. Entangling single- and N -atom qubits for fast quantum state detection and transmission. Phys. Rev. A, 72:042302, 2005.

[81] B. C. Jacobs and J. D. Franson. All-optical switching using the quantum Zeno effect and two-photon absorption. e-print arXiv:quantph/0905.1261v1, 2009.

[82] H. You, S. M. Hendrickson and J. D. Franson. Analysis of enhanced two-photon absorption in tapered optical fibers. e-print arXiv:quantph/0810.0016v1, 2008.

[83] T. Nakanishi, H. Kobayashi, K. Sugiyama and M. Kitano. Full quantum analysis of two-photon absorption using two-photon wavefunction: Comparison with one-photon absorption. e-print arXiv:quantph/0906.0213v1, 2009.

[84] K. Singer, M. Reetz-Lamour, T. Amthor, L. G. Marcassa and M. Weidemüller. Suppression of excitation and spectral broadening induced by interactions in a cold gas of Rydberg atoms. Phys. Rev. Lett., 93:163001, 2004 . 
[85] J. Deiglmayra, M. Reetz-Lamour, T. Amthora, S. Westermanna, A. L. de Oliveira and M. Weidemüllera. Coherent excitation of Rydberg atoms in an ultracold gas. Optics Communications, 264:293, 2006.

[86] S. Quabis, R. Dorn, M. Eberler, O. Glöckl and G. Leuchs. The focus of light - theoretical calculation and experimental tomographic reconstruction. Appl. Phys. B, 72:109, 2001.

[87] S. J. van Enk. Atoms, dipole waves, and strongly focused light beams. Phys. Rev. A, 69:043813, 2004.

[88] M. K. Tey, Z. Chen, S. A. Aljunid, B. Chng, F. Huber, G. Maslennikov and C. Kurtsiefer. Strong interaction between light and a single trapped atom without the need for a cavity. Nature Physics, 4:924, 2008.

[89] M. K. Tey, G. Maslennikov, T. C. H. Liew, S. A. Aljunid, F. Huber, B. Chng, Z. Chen, V. Scarani and C. Kurtsiefer. Interfacing light and single atoms with a lens. New J. Phys., 11:043011, 2009.

[90] M. Sondermann, R. Maiwald, H. Konermann, N. Lindlein, U. Peschel and G. Leuchs. Design of a mode converter for efficient light-atom coupling in free space. Appl. Phys. B, 89:489, 2007.

[91] J. Wei, D. Olaya, B. S. Karasik, S. V. Pereverzev, A. V. Sergeev and M. E. Gershenson. Ultrasensitive hot-electron nanobolometers for terahertz astrophysics. Nature Nanotechnology, 3:496, 2008.

[92] R. Raussendorf, J. Harrington and K. Goyal. A fault-tolerant one-way quantum computer. Ann. Phys., 321:2242, 2006.

[93] A. Y. Kitaev. Fault-tolerant quantum computation by anyons. Ann. Phys., 303:2, 2003.

[94] R. Raussendorf, J. Harrington and K. Goyal. Topological faulttolerance in cluster state quantum computation. New J. Phys., 9:199, 2007.

[95] T. M. Stace, S. D. Barrett and A. C. Doherty. Thresholds for topological codes in the presence of loss. Phys. Rev. Lett., 102:200501, 2009.

[96] W. Dür, H. Aschauer and H. J. Briegel. Multiparticle entanglement purification for graph states. Phys. Rev. Lett., 91:107903, 2003. 
[97] H. Aschauer, W. Dür and H. J. Briegel. Multiparticle entanglement purification for two-colorable graph states. Phys. Rev. A, 71:012319, 2005 .

[98] P. G. Kwiat, E. Waks, A. G. White, I. Appelbaum and P. H. Eberhard. Ultrabright source of polarization-entangled photons. Phys. Rev. A, 60:R773, 1998. 